NBER WORKING PAPER SERIES

\title{
DYNAMIC EFFECTS OF CO-ETHNIC NETWORKS ON IMMIGRANTS' ECONOMIC SUCCESS
}

Michele Battisti

Giovanni Peri

Agnese Romiti

Working Paper 22389

http://www.nber.org/papers/w22389

\author{
NATIONAL BUREAU OF ECONOMIC RESEARCH \\ 1050 Massachusetts Avenue \\ Cambridge, MA 02138 \\ July 2016, Revised May 2018
}

The authors would like to thank seminar participants at the Ifo Institute in Munich, University of Arizona, University of Innsbruck, University of Passau, University of Bergen, University of Bielefeld, Copenhagen Business School, IAB Nuremberg, DIW, as well as conference participants at the 2016 AIEL, 2016 IZA Annual Migration Meeting, 2016 EALE, 2016 ESPE and 2015 SAEe Conferences. Michele Battisti gratefully acknowledges financial support for this project by the Leibniz Association (SAW-2012-ifo-3). The views expressed herein are those of the authors and do not necessarily reflect the views of the National Bureau of Economic Research.

NBER working papers are circulated for discussion and comment purposes. They have not been peer-reviewed or been subject to the review by the NBER Board of Directors that accompanies official NBER publications.

(C) 2016 by Michele Battisti, Giovanni Peri, and Agnese Romiti. All rights reserved. Short sections of text, not to exceed two paragraphs, may be quoted without explicit permission provided that full credit, including $(\odot)$ notice, is given to the source. 
Dynamic Effects of Co-Ethnic Networks on Immigrants' Economic Success

Michele Battisti, Giovanni Peri, and Agnese Romiti

NBER Working Paper No. 22389

July 2016, Revised May 2018

JEL No. J24,J61,R23

\section{ABSTRACT}

This paper investigates how the size of co-ethnic networks at the time of arrival affect the economic success of immigrants in Germany. Applying panel analysis with a large set of fixed effects and controls, we isolate the association between initial network size and long-run immigrant outcomes. we also look at those who were assigned to an initial location independently of their choice allows a causal interpretation of our estimates. We find that immigrants initially located in places with larger co-ethnic networks are more likely to be employed at first, but have a lower probability of investing in human capital.

Michele Battisti

Ifo Institute

Poschingerstraße 5

81679 Munich

Germany

battisti@ifo.de

Giovanni Peri

Department of Economics

University of California, Davis

One Shields Avenue

Davis, CA 95616

and NBER

gperi@ucdavis.edu
Agnese Romiti

IAB Institute for Employment Research

agnese.romiti@iab.de 


\section{Introduction}

The labor market assimilation and the economic integration of new immigrants are crucial to their overall success and to that of the host country. What is the role of other immigrants from the same country of origin in determining such economic success? Do new immigrants benefit from their presence when looking for jobs and careers? Or are new immigrants constrained by them, as these networks limit them to informal channels missing the larger labor market and, possibly, discouraging them from the acquisition of general human capital? How do the effects differ between the short run and the long run? This paper answers these questions using survey data on recent immigrants to Germany from the IAB-SOEP Migration Sample matched with the universe of administrative records of the German social security archive (Integrierte ErwerbsBiografie or IEB). The merged dataset, which is very novel, includes pre-migration information on individual migrants and follows them after arrival in Germany collecting yearly information on labor market, demographic and education variables. Our findings inform whether policies encouraging immigrants concentration or those encouraging dispersion are more conducive to short and longrun success in the labor market.

The causal effect of the size of the co-ethnic network on immigrants' labor market success is not easy to assess. The main challenge is that the size of the co-ethnic network (i.e. the network of individuals from the same country of origin) itself affects the type of immigrants drawn to the area and it is therefore likely to be correlated with observable and unobservable characteristics. Comparing post-migration outcomes of new immigrants between areas with large and small co-ethnic networks would imply comparing individuals that are systematically different. New immigrants tend to cluster where co-ethnic immigrants are already settled both in the US (Cutler and Glaeser, 1997, and Borjas, 1998), and in Germany (Glitz, 2014) and this tendency may vary across ethnicity and with immigrants characteristics. For instance, using social security data for Germany in 2008, Glitz (2014) computes measures of segregation and finds that Western Europeans ${ }^{1}$ and Turks were the groups with the highest segregation indices. He also finds that less educated immigrants were more segregated than more educated ones. Since these and other unobserved characteristics are correlated with the economic success of immigrants, OLS analysis will not identify correctly the causal effect of the local co-ethnic network.

\footnotetext{
${ }^{1}$ Excluding Italy and Greece.
} 
In order to think more systematically about the role of co-ethnic networks in affecting the short and long-run employment and wage outcomes of new immigrants, we discuss a simple theoretical framework. A partial equilibrium search model illustrates the trade-off between employment and human capital investment after arrival in the destination country. We consider workers receiving employment offers through a formal search channel and an informal/network-based channel. How effective the latter is depends on the size of the local co-ethnic network. On the other hand, the effectiveness of the formal search method is affected by one's stock of human capital. Job opportunities have a distribution of wages that can be different between formal and informal offers and they are accepted if they pay more than the reservation wage. The key predictions of our model are that, while large co-ethnic networks have a positive effect on the chances of finding employment after arrival, over time immigrants who started in locations with small co-ethnic networks catch up and may have similar or higher employment probabilities and higher wages. The closing of the employment gap is due to the higher human capital investment of new immigrants in markets with small initial co-ethnic networks. For them, the incentive to increase their general human capital is higher and the cost is lower in the short run, because the opportunity cost of the foregone job search is smaller. Therefore, our model suggests that it is important to distinguish between short-run and long-run impacts of co-ethnic networks on employment, wages and human capital. This distinction has not received much attention in the literature, partly because of the lack of direct measures of human capital investment by immigrants, and partly because of a very limited availability of panel datasets following immigrants. As long as human capital investments are important for the economic assimilation of immigrants, the long-run focus of this analysis is key to set up effective policies.

We then investigate whether these simple predictions hold empirically. We are contributing to the literature in three ways. First, we estimate the dynamic (short and long-run) effects of the size of the co-ethnic network at arrival on new immigrants' employment by taking advantage of the panel nature of our dataset. ${ }^{2}$ Second, we analyze the investment in human capital of new immigrants after arrival as an additional outcome. This is a crucial margin to understand the differences in outcomes between the short and long run. To the best of our knowledge, there is no other study that investigates the role of co-ethnic networks on human capital investment

\footnotetext{
${ }^{2}$ To our knowledge Edin et al. (2003) is the only study shortly mentioning the dynamics of the network effect, though the paper focuses entirely on the static mechanism.
} 
of first-generation immigrants after their arrival in the destination country. ${ }^{3}$ Third, thanks to the novel survey data, we have direct information on job search methods and in particular on whether people have found jobs through personal contacts or through market/agency/Internet search. Hence, this study is one of the rare cases in which we can directly check the importance of a "network" channel in finding a job and test if the predictions align with those of the model. ${ }^{4}$

As mentioned above, the identification of a causal effect of network size on new immigrants' outcomes is difficult: the endogenous sorting of new immigrants across locations along observable and unobservable characteristics poses a big challenge. Location decisions depend on individual characteristics that may affect post-migration labor market outcomes. A first approach used in the literature for reducing the selection bias is measuring co-ethnic networks at a relatively broad local level. As pointed out by Bertrand et al. (2000), Cutler and Glaeser (1997) and Dustmann and Preston (2001), immigrants' location decisions are affected by the presence of previous immigrants in the specific district of residence (typically a city block), but much less by their presence in the larger region (local labor market). Still that local presence of co-ethnic networks helps job connections and is used as explanatory variable. This strategy is helpful, but does not fully address the problems of endogenous sorting. Recent papers, including Edin et al. (2003) and Damm (2009), have exploited a different strategy. Researchers have noticed that national and international dispersal policies, usually applied to refugees, have generated an exogenous initial location of refugees. These policies, by distributing individuals independently of their skills and labor characteristics, have generated quasi-experimental variation in the initial exposure to co-ethnic networks, which could be used to identify a causal effect on later outcomes. Limiting the attention to refugees makes this type of identification credible. However, this group is very different from the rest of the immigrant population, limiting the external validity of such an exercise. ${ }^{5}$ Refugees come from traumatic situations, often experience periods of non-employment before migration, and come from specific countries. This might not correspond to the experience of other immigrants, usually attracted by family and employment opportunities. Hence the

\footnotetext{
${ }^{3}$ Investments in schooling and education are mentioned in other studies (for example in Edin et al. (2003) and Damm (2009)) as possible channels through which networks have an effect. They have never been studied directly, however, because of data limitations.

${ }^{4} \mathrm{~A}$ rare study analyzing the channels through which people find jobs and relating them to network size is Dustmann et al. (2016), where the network is defined at the firm level.

${ }^{5}$ Table D.1 in Appendix D shows that in our dataset these differences are substantial.
} 
network effects on refugees may not be representative of those on other immigrants.

Our approach improves on these methods for two reasons. First, the survey data provide us with pre-migration characteristics of immigrants such as employment status, work experience, education level, language proficiency, and cognitive abilities before arrival. This allows us to control for several relevant characteristics (considered as unobservable in previous studies) and to test how these pre-migration characteristics are correlated to their initial location and in particular to the size of the local co-ethnic network at arrival. Second, as our data include different national groups in different cities over time we can control for nation-level fixed effects that should absorb all the common traits from a group and for city-year fixed effects that absorb local economic conditions. Third, we can identify in our sample those individuals who were subject to dispersal policies (refugees and ethnic Germans in this case). By doing so we can evaluate how the estimated effects for all immigrants compare with those on the randomly dispersed group of refugees and ethnic Germans, after including fixed effects and pre-migration characteristics. This allows us to assess the external validity of effects estimated on a restricted group and how effectively we corrected for omitted variable and selection bias, using fixed effects and controls, in the sample of all immigrants. Similar estimates using either method would significantly reduce worries of selection and omitted variable bias.

Our three main empirical findings support the key predictions of the model. First, we find that immigrants in districts with larger initial co-ethnic networks are significantly more likely to find employment within their first three years in Germany. Second, we find that this advantage fades away in the longer run and it is not present after around five years. Third, the likelihood that immigrants carry out human capital investments within three years since migration decreases with the size of co-ethnic network at arrival. As general human capital investment improves the opportunities in the labor market (in terms of wage and employment), the initial advantage in employment probability due to large-networks fades away over time.

Even when controlling for district-year, origin-year, and origin-district fixed effects to absorb local economic effects, and controlling for individual pre-migration characteristics, we find significant positive short-run effects of the size of co-ethnic network on employment probability and negative short-run effects on human capital investment. In addition, we find that, in the long-run, the advantage in employment probability disappears and immigrants with smaller initial co-ethnic network have higher wages, possibly because of larger investments in human capital and better 
match quality. Immigrants with smaller initial co-ethnic networks are also less likely to find their job through referrals. These effects are largely driven by less educated immigrants, while for those with tertiary education the size of initial network does not seem to affect economic outcomes. Finally, when we restrict our analysis to the sample of refugees and ethnic Germans subject to dispersal policies, ${ }^{6}$ we find similar effects of initial network on initial employment probability and on human capital investments, which suggests that our panel estimates do not suffer from omitted variable and selection bias. We also perform a series of robustness checks and falsification exercises, including a different definition of the geographic level at which we measure networks, a placebo-type exercise where we address possible concerns of the networks being a proxy for local demand fluctuations. These exercises confirm our main results and the validity of our identification strategy.

The rest of the paper is organized as follows. In Section 2 we review the related literature in relation to our contribution. In Section 3 we present our theoretical setup. Section 4 describes our data sources and presents some summary statistics; Section 6 discusses identification, estimating specification and results, including robustness checks and test for the determinants of initial location. Section 7 concludes.

\section{Literature Review}

Our paper is related to research on the effects of networks on job search and labor market outcomes. Much of this literature does not analyze immigrants per se, but focuses on the role of social networks on economic outcomes in general. Important theoretical contributions to the modeling of social networks and their effects on the labor market build on Calvó-Armengol and Jackson (2004). Beaman (2012) develops a network model with multiple cohorts to investigate the relative importance of information transmission and competition in networks and their consequences on the labor market. Bayer et al. (2008) investigate the effect of living in the same city block on the likelihood of working together, finding an important role for referrals in the labor market. Goel and Lang (2009) show that networks may bring about additional job offers, thereby raising the observed wages of workers in jobs found through formal channels relative to those in jobs found through the network. ${ }^{7}$ Our model, which

\footnotetext{
${ }^{6}$ See section B in the Appendix for the institutional background. Glitz (2012) also uses this group exploiting its random allocation as a source of exogenous variation in labor supply.

${ }^{7}$ Pellizzari (2010) also develops a search model with a formal and an informal channel and match specific productivity.
} 
builds upon Goel and Lang (2009), combines a simple search model with the choice of human capital investment. Several papers frame networks as alternative to search in the general labor market. The network provides an advantage in the probability of a match but it may be limited by the specificity and cost of referrals. Galenianos (2013, 2014) develop models where network and formal market coexist and different individuals use either of them depending on relative costs and benefits. Our model can be seen as a simple case within this line of inquiry.

As mentioned above, a number of papers use the initial dispersal of refugees across locations to achieve empirical identification of the effect of the co-ethnic networks on labor market outcomes. Edin et al. (2003) use data from a dispersal policy in Sweden and find positive effects of network size on earnings for less skilled immigrants. They also point out that networks might have a positive effect on information and a negative effect on human capital acquisition. However, they are not able to investigate the empirical importance of that channel because their data do not include any measure of human capital investment. Similarly, Damm (2009) investigates the effects of ethnic enclaves on labor market outcomes in Denmark by taking advantage of a dispersal policy and finds a large positive static effect of ethnic enclaves on earnings after migration. Xie and Gough (2011) analyze the role of ethnic enclaves on labor market outcomes in the US, and find no evidence of a positive effect of ethnic enclaves on earnings of new immigrants. However, the analysis is mainly based on correlations. Hellerstein et al. (2011) look at the role of residential proximity on the chances that workers work at the same establishment. ${ }^{8}$

Recently, interesting work looks at the role of referrals on employment outcomes at the firm level. Dustmann et al. (2016) develop a model of job referrals by which current employees in a firm provide information on potential candidates, and test the main predictions of the model using information on ethnic origin of employees of a large metropolitan market in Germany. They find that firms tend to hire workers from ethnic groups that are already represented in the firm and that hiring through referrals pay higher wages and exhibits lower turnover. This suggests that referrals may improve the quality of employer-employee matches. Similarly, Patacchini and

\footnotetext{
${ }^{8}$ Using Danish administrative data, Bennett et al. (2015) look at the role of attitudes as well as networks on educational attainments of teenagers with a migration background. Åslund et al. (2011) analyze the role of neighborhood characteristics on the school performance of immigrant children, using data from an exogenous refugee policy in Sweden. Using the mass migration wave to Israel as exogenous variation, Gould et al. (2009) look at the effects of high exposure to immigrants during elementary school on the long-term educational attainment of natives.
} 
Zenou (2012) analyze the effect of ethnic networks on job search methods, and find results that confirm a positive role of networks on the probability of finding a job through referral. Analysis from our survey confirms these findings.

We believe that our paper has several original features, relative to the previous literature. First, our combination of data on the pre- and post-migration history of individual workers is novel. Then the measures of co-ethnic network in the place of arrival, based on the universe of immigrant workers, is also more precise and detailed than that of other studies, enabling panel analysis with a richer set of fixed effects. Finally, the possibility of comparing all immigrants to a group of immigrants whose initial location was determined by dispersal is also unique. Controlling for premigration features of immigrants and local economic conditions is important for the identification of the effects of interest, and using all immigrants (rather than refugees only) is important for external validity. We also perform an analysis of the dynamic effects of networks from arrival throughout the working career of immigrants, and include for the first time the analysis of investment in human capital.

\section{Theoretical Framework}

The main goal of our very simple framework, which builds upon Montgomery (1991) and Goel and Lang (2009), is to illustrate the trade-off between search and human capital investments, which provides the key insight for our empirical predictions. Let us consider two periods, $t=1,2$. At the beginning of $t=1$ the agent (a newlyarrived immigrant) enters the local economy with a certain level of human capital, which we take as exogenous. The level of human capital in periods 1 and 2 are denoted by $h_{1}$ and $h_{2}$. We interpret human capital as the general set of skills that are valued in the host country labor market. The initial value of $h$ is determined by its pre-migration level and its transferability. The size of co-ethnic network at the initial location is denoted as $n_{1}$. We denote a certain realization of $h$ by $\bar{h}$ and a certain realization of $n$ by $\bar{n}$. The first period is the arrival period in the destination country, and we assume that all individuals are initially unemployed.

There are two mechanisms through which workers receive job offers. ${ }^{9}$ First, when searching for a job, there is a certain probability that the worker receives

\footnotetext{
${ }^{9} \mathrm{~A}$ more general model is van den Berg and van der Klaauw (2006), where the intensity of the search is endogenous. For simplicity in our model the only endogenous choice is whether to search or to invest in human capital during the first period.
} 
an offer through the formal channel. ${ }^{10}$ We denote this probability by $p_{f}$ and we assume that it depends positively on the human capital level of the individual, so that $\partial p_{f}(h) / \partial h>0$, and that it does not depend on the size of the local network. The individual may also receive an offer from the co-ethnic network channel (or informal channel) with a probability $p_{i}$, which depends positively on the size of the co-ethnic network, such that $\partial p_{i}(\bar{n}) / \partial n>0$ and does not depend on the individual's human capital. We assume decreasing marginal returns for both channels, i.e. $\partial^{2} p_{i}(n) / \partial n^{2}<0$ and $\partial^{2} p_{f}(h) / \partial \bar{h}^{2}<0 .{ }^{11}$

At the beginning of each period, the worker decides whether to search for a job or to invest in general human capital, engaging in activities that increase her human capital level $h$. If the individual looks for a job, she has some chances of getting an offer from either channel, as outlined above. We do not need to assume that wages are drawn from the same wage offer distribution in the formal and network channel. We restrict, however, wages drawn from either distribution to be always positive, we assume the draws to be independent and that the two wage offer distributions have overlapping support. ${ }^{12}$ For convenience, we assume that those distributions do not change between period 1 and period 2. We denote the common cumulative distribution of wage offers obtained in the formal channel as $F_{f}(w)$. Correspondingly, wage offers in the network channel are drawn from $F_{i}(w)$. Instead of searching for a job, the individual can increase her human capital endowment. Her human capital after education is $\bar{h}^{\prime}>\bar{h}$. We assume that $\bar{h}^{\prime}=\bar{h}+A$ where $A$ is a positive quantity. This is equivalent to assuming that human capital increase is independent from its initial level. Combined with $\partial^{2} p_{f}(h) / \partial \bar{h}^{2}<0$, this assumption implies that investing in education has larger marginal effects on labor market perspectives of individuals with low initial levels of human capital. At the beginning of period 2, an agent that has chosen human capital investment in the previous period is more likely to get offers through the formal channel, and therefore less likely to be unemployed, and has a higher expected wage (because of the possibility of receiving two offers).

The key decision for the agent is made at the beginning of period 1. If she searches

\footnotetext{
${ }^{10}$ One characterization of the "formal channel" would be a matching mechanism where applicants send applications with their resumes to employers or to an employment agency.

${ }^{11}$ Since $p_{i}$ and $p_{f}$ are probabilities, they are bounded between zero and one. However, we are not imposing the constraint that $p_{f}+p_{i}=1$. This is because in our model an individual searching for a job can get either zero, one or two offers.

${ }^{12}$ This means that the highest possible offer from one of the two distributions cannot be lower than the lowest offer from the other distribution. In that case, there would be no gain in drawing two offers instead of one. That is a case we can deal with, but believe that it is not of interest.
} 
for a job, she will receive an offer through the formal channel with probability $p_{f}(\bar{h})$, and an offer through the network channel with probability $p_{i}(\bar{n})$. If she receives no offer, she remains unemployed, receives unemployment payments $b_{u}$ and begins period 2 with the same level of human capital $h_{2}=h_{1}=\bar{h}$. If she receives one offer, from either channel, she will accept it if higher than $b_{u}$ and reject it otherwise. We assume $b_{u}$ to be time invariant and that the agent gets no utility from leisure, so the decision in the second period is equivalent to the one in the first period. If the agent receives two offers, she will accept the higher offer if it is higher than $b_{u}$, and reject both otherwise. If the individual decides to get education instead, she receives $b_{h}$ in period 1 with certainty, and will have a higher level of human capital $h_{2}=\bar{h}^{\prime}>\bar{h}$, in period 2. This allows her to have more chances to receive an offer from the formal channel at $t=2$. We assume that $b_{u} \geq b_{h}$ to allow for some costs of education. ${ }^{13}$

\subsection{Preferences}

Our agent values consumption and discounts second period outcomes at the rate $0<\beta<1$. We assume utility to be linear in consumption ${ }^{14}$

$$
E U\left(c_{1}, c_{2}\right)=c_{1}+\beta E\left(c_{2}\right)
$$

As a standard two-period model, the solution is best described using backward induction. We start by illustrating possible payoffs at period 2. At $t=2$, human capital investment will not occur since $b_{u} \geq b_{h}$. Therefore, the individual will search for a job at $t=2$ for all realizations of the exogenous parameters. If the agent acquired human capital in period $t=1$, she will be able to search for a job with a higher probability of receiving an offer through the formal channel, and therefore also a higher probability of receiving two offers. If the agents searched in period 1 , she will search again with the same human capital endowment as in $t=1$. $^{15}$

\footnotetext{
${ }^{13}$ While this assumption seems natural in this context, it is stronger than needed in our model, as we only need to assume that expected income is larger for those who look for a job at $t=2$. None of the main propositions discussed below depend on this assumption.

${ }^{14}$ Implicitly we are assuming that individuals are endowed with one unit of "effort" (or time) each period and supply it to education or search/work.

${ }^{15} \mathrm{We}$ assume separation rates at the end of each period to be equal to one so that our problem is recursive. None of our qualitative results depends on this assumption. We are not investigating the possibility that employment can generate human capital as well. As long as the growth in human capital is smaller when working than when in school, the main results of our model are robust to relaxing this assumption.
} 


\subsection{Value functions}

At the beginning of $t=2$, all individuals search for a job. If the agent has searched at $t=1$ (whether or not she found a job in that period) then $h_{2}=h_{1}=\bar{h}$, and her expected payoff from searching in period 2 is

$$
\begin{aligned}
S_{2}(\bar{n}, \bar{h}) & =b_{u}+p_{i}\left(1-p_{f}\right) \int \max \left\{W_{2}\left(x_{i}\right)-b_{u}, 0\right\} d F_{i}\left(x_{i}\right) \\
& +p_{f}\left(1-p_{i}\right) \int \max \left\{W_{2}\left(x_{f}\right)-b_{u}, 0\right\} d F_{f}\left(x_{f}\right) \\
& +p_{i} p_{f} \int \max \left\{W_{2}\left(x_{i}\right)-b_{u}, W_{2}\left(x_{f}\right)-b_{u}, 0\right\} d F_{i}\left(x_{i}\right) d F_{f}\left(x_{f}\right) \equiv \mathcal{S}(\bar{n}, \bar{h})
\end{aligned}
$$

where we omitted the dependence of $p_{i}$ and $p_{f}$ on network size $\bar{n}$ and human capital $\bar{h}$. Searching in period 2 means the agent gets at least $b_{u}$, and has a certain probability to receive wage offers that are higher than $b_{u}$. The agent may instead enter period 2 after having invested in human capital in period $t=1$. In this case her human capital is $\bar{h}^{\prime}>\bar{h}$ and therefore the value of searching is $\mathcal{S}\left(\bar{n}, \bar{h}^{\prime}\right)>\mathcal{S}(\bar{n}, \bar{h})$ because of our assumption that $\partial p_{f} / \partial h>0$ (under this assumption $\partial S_{2} / \partial p_{f}>0$ ).

At the beginning of period 1 the agent decides whether to make an educational investment or to search for a job immediately, and will do so taking account of how that choice affects outcomes at $t=2$. If the agent decides to search for a job in period $t=1$ given an initial network size of $\bar{n}$ and initial human capital level $\bar{h}$ the value function can be simply written as:

$$
S_{1}(\bar{n}, \bar{h})=\mathcal{S}(\bar{n}, \bar{h})+\beta \mathcal{S}(\bar{n}, \bar{h})=(1+\beta) \mathcal{S}(\bar{n}, \bar{h})
$$

A searching individual gets the value of being unemployed plus the possible gain from employment. At the beginning of period 1 the individual may instead decide to invest in human capital. The corresponding value function is

$$
H_{1}(\bar{n}, \bar{h})=b_{h}+\beta \mathcal{S}\left(\bar{n}, h^{\prime}\right)
$$

Costs of education are incorporated in $b_{h} \cdot{ }^{16}$ Education increases future employment possibilities as the newly acquired skills are useful to find a job in the host economy. Therefore, the lower employment prospects are and the higher the discount rate $(\beta)$ is, the more likely it is that an agent invests in human capital at $t=1$.

\footnotetext{
${ }^{16}$ Results may be different for a risk-averse agent since returns to education are stochastic.
} 


\subsection{Employment and Human Capital Investment}

The simple structure described above is sufficient to illustrate the main trade-off faced by the agent. Human capital investment increases employment and expected wages in the future, at the cost of foregoing current earnings. After observing her level of human capital and the size of the social network at the beginning of period 1 , the individual decides whether to look for a job or to acquire human capital. The optimal decision between searching and acquiring human capital will be given by comparing $S_{1}(\bar{n}, \bar{h})$ and $H_{1}(\bar{n}, \bar{h})$. Next, we discuss how this optimal choice depends on the initial level of $\bar{n}$ and $\bar{h}$. We are able to make three simple predictions in a comparative statics exercise.

Proposition 1 For each level of $n_{1}$ there is at most one "reservation" level of $h_{1}$ below which the agent will invest in human capital and above which the agent will search for a job in period 1. See Appendix A for discussion.

For a given level of $n_{1}$, both the value of searching and the value of investing in human capital are increasing, concave functions of $h_{1}$. Under our assumptions, the relative first and second derivatives are such that the two curves $S_{1}(\bar{n}, h)$ and $H_{1}(\bar{n}, h)$ will intersect at most once in the $h$ space. ${ }^{17}$ Depending on functional form and support of $h$ and $n$, corner solutions may exist: initial social networks $n$ may be so large that the agent may find it optimal to search for a job irrespective of the level of $h$. Following Proposition 1, for a given level of social networks, individuals with higher human capital are more likely to be employed in period 1 and less likely to invest in further human capital. Individuals with lower human capital are more likely to get education and less likely to be employed soon after arrival.

Proposition 2 For each level of $h_{1}$ there is at most one "reservation" level of $n_{1}$ below which the agent will invest in human capital and above which the agent will search for a job in period 1. See Appendix A for discussion.

For a fixed value of $h_{1}=\bar{h}, S_{1}(n, \bar{h})$ is increasing in the level of $n_{1}$, because $n_{1}$ positively affect offers' arrival rate via the network channel. It is only slightly more subtle to see why the value of human capital investment is lower at higher values of $n_{1}$. Let us imagine a case in which an individual with a very large social network decided to acquire further education in period 1. Despite the higher level of

\footnotetext{
${ }^{17}$ We analyze the two functions $S_{1}$ and $H_{1}$ in more detail below and in Appendix A.
} 
human capital, it would still be relatively likely for her to get an offer in the informal sector compared to the formal sector, and therefore for her further human capital investment makes less of a difference. Corner solutions may exist in this case as well: there might be levels of human capital that are high enough such that the agent searches for a job in period 1 for any possible level of social networks. Proposition 2 implies that individuals with larger co-ethnic networks are less likely to get further education and more likely to be employed in the first period.

Proposition 3 The magnitudes of the effects of networks on employment and human capital investment are lower if the individual has a higher initial human capital endowment. See Appendix A for discussion.

Individuals with higher initial human capital endowment $h_{1}$ are relatively more likely to find a job through the formal channel compared to individuals with the same networks but with lower initial human capital endowment. The marginal effect of network size in the value functions of individuals with initially high human capital is therefore going to be smaller. While qualitative effects of network size are unaffected, effects on employment are quantitatively larger for individuals with lower initial human capital endowments. ${ }^{18}$

Summarizing, based on our model we expect individuals with larger initial coethnic networks to be more likely to find employment after arrival. However, our model also predicts the positive effect of a co-ethnic network on employment probability to decrease over time, because individuals with smaller co-ethnic networks "catch up" through human capital investment. Finally, the effect of network size on employment probability and on human capital investment after immigration are larger for individuals with lower initial human capital. Figure 1 summarizes the main features of the equilibrium of our model. It plots the value functions of an individual, $S_{1}$ and $H_{1}$, as a function of initial network size. An individual with lower initial human capital $\underline{h}$ will optimally decide to invest in human capital if her initial network size is below $n_{\underline{h}}$, and she will search for a job if it is larger. This illustrates

\footnotetext{
${ }^{18}$ In order to make predictions concerning whether we expect individuals with low initial human capital or individuals with high initial human capital to be more likely to invest in it, we need to give some structure to the returns to human capital. If returns to human capital are smaller for individuals with high initial human capital endowment, which is the standard assumption in the literature and has support in our data, individuals with lower initial human capital are more likely to invest in its improvements. Results would change if returns to human capital were larger for individuals with larger initial stocks. This case would be closer to Regets and Duleep (1999).
} 
Proposition 2 above. The two thicker curves in Figure 1 are instead drawn for an individual with higher human capital $n_{\bar{h}}>n_{\underline{h}}$. Both $S_{1}$ and $H_{1}$ are higher (because at higher human capital levels the expected utilities are higher due to higher probability of job offers) and flatter (reflecting the fact that marginal effects of network size are smaller at higher levels of human capital, because offers are more likely to come from the formal channel, making networks less relevant for labor market outcomes as in Proposition 3). The new threshold for network size below which the individual invests in human capital is now lower at $n_{\bar{h}}$, because the shift of the value function for search is larger than that of the value function for human capital investment. ${ }^{19}$ This shift from $\underline{h}$ to $\bar{h}$ is an illustration of Proposition 1 above. The figure shows a range of intermediate network sizes for which individuals with lower levels of initial human capital invest, while individuals with higher levels of initial human capital search for a job in the first period.

\section{$3.4 \quad$ Wages}

In the paragraphs above, we have discussed the implications of our model for employment and human capital investment. Next, we look at the effects on observed wages, i.e. wages of those who are employed. Even if the distributions of wages from each channel (market and network) are given, the realized wage of an individual depends on the probability of getting competing offers. When an individual has a higher chance of receiving two offers, she also has a larger expected wage, but may not have a higher observed wage. Therefore, without additional assumptions on the wage distributions of the two channels, our model cannot deliver any predictions on relative observed wages at $t=1$, because more chances to draw from a distribution can lower observed wages of the employed. For the analysis below, we therefore further assume that the wage offer distribution of the formal channel and of the network channel have the same expected value. This rules out that a higher probability of receiving an additional offer depresses average wages. Under this assumption, observed wages at $t=1$ are a monotonically increasing function of $n$ : conditional on $h_{1}$, a higher $\bar{n}$ increases the likelihood of receiving two offers, which is associated with a higher expected wage (although this effect may be weak).

The relationship between initial network size and observed wages at $t=2$ is slightly more complicated. Assuming that initial human capital is low enough that

\footnotetext{
${ }^{19}$ We discuss the details of this in our Appendix.
} 
an internal solution exists, at low levels of $n_{1}$, the individual will acquire human capital and enter period 2 with $h_{2}>h_{1}$. Observed wages at time $t=2$ are increasing in $n_{1}$ because larger social networks increase the probability of receiving two offers. However, this effect exhibits a discontinuity at the level of social networks above which the individual does not invest in human capital at $t=1$. If $n_{1}$ is high enough the individual will not find it profitable to invest in human capital at $t=1$, then her wages at $t=2$ will be lower. For changes in initial network size that are large to matter for human capital accumulation decisions, individuals with larger network are expected to have lower wages in the long run. Figure 2 depicts the relationship between the wage in the second period and the size of the network, graphically. ${ }^{20} \mathrm{As}$ for the previous result, we expect this effect to be concentrated among those with relatively low initial human capital, for whom initial network size is more likely to matter for human capital decisions.

\subsection{Networks and Welfare}

Describing the welfare implications of different distributions of networks in the society is beyond the scope of our work. However, a brief discussion on the way networks may matter for both individual and social welfare may be useful. In our simple model, networks may induce people to invest less in human capital. However, that choice is optimal at the individual level and - with rational, forward looking agents and no externalities - also maximizes utilitarian social welfare. On the other hand, there are realistic scenarios under which this may not be the case and where larger networks may hurt social welfare, while increasing individual welfare. First, if we introduced progressive taxation, returns to education at the individual level would be lower than at the level of the society as a whole. Alternatively, if individual migrants discount the future more than the social planner, or if education generates positive externalities, agents may be underinvesting in general human capital (from the perspective of the social planner) and this underinvestment would be more severe when there are large networks and for less educated people. In these cases, there would be an economic rationale for a government intervention to encourage immigrants to distribute across locations, or that encourages searching via the formal channel (where there may be limited opportunities for immigrants) rather than via networks.

\footnotetext{
${ }^{20}$ Figure 2 is drawn under the assumption that initial human capital is low enough to make human capital investment at $t=1$ optimal for low enough $n$.
} 


\section{Data}

Our primary dataset is the IAB-SOEP Migration Sample, a large survey of immigrants to Germany conducted in two waves that took place in 2013 and 2014. The survey oversamples immigrants who arrived in Germany after 1994. We use the subsample of the survey that has been linked to the social security data (which includes all workers covered by the social security system, excluding civil servants, self-employed, and military personnel) selecting only foreign-born people aged 15$65 .{ }^{21}$ We are able to observe several individual pre-migration characteristics, as well as the entire labor market history after migration to Germany. The data on employment and wages are from IEB and cover the period 1975-2013. ${ }^{22}$ Wages are measured as average real hourly wage of all full-time working spells in the year excluding apprenticeship, or marginal employment. Our measure of human capital investment originates from the survey data. The survey provides a full account of each year spent in education as each individual is asked retrospectively to fill a life-long calendar and to report for each year, starting from age 15, whether in that year she was in education. ${ }^{23}$ We use this information to reconstruct an individual life-long panel of spells of education and we merge this to the individual administrative records. ${ }^{24}$

The variable capturing the co-ethnic network size at arrival for each immigrant is calculated as the number of workers by nationality ${ }^{25}$ as share of total employment in each district in the year in which the immigrant first arrives to Germany. ${ }^{26}$ This share is calculated using the full registry of employees in Germany (IEB). The number of German districts is 404, with an average size of 65,801 workers per district and a median size of 42,643. Our sample of immigrants is distributed across 229 districts.

\footnotetext{
${ }^{21}$ The survey is targeted at individuals with any migration background, including second generation immigrants. See Appendix C for details.

${ }^{22} \mathrm{~A}$ person is considered employed if she ever works within the year. We check the robustness of the results to this criterion by using alternative definitions of employment, see Table D.5. The results are robust to defining an individual as employed if she/he works at least 25,50 , or 75 percent of the year, or at a cut-off date.

${ }^{23}$ Unfortunately, we cannot distinguish the type of education, but, given our selected age range, we argue that school episodes play a minor role. In addition, we exclude individuals that are in school at the time of the survey and individuals that entered Germany as students.

${ }^{24}$ To limit recall bias, we also use administrative data, setting the variable to zero if the person in the corresponding year works for at least 50 percent of the time.

${ }^{25}$ Due to sample size considerations, we group them into eight country groups: Western countries including Western Europe, Eastern Europe, South-Eastern Europe, Turkey, USRR, Asia and Middle East, Africa, Central and South America.

${ }^{26}$ We define the living district as the one corresponding to the longest spell within the year, and impute this information from the workplace district in case of missing values.
} 
Our network measure has an average size of 0.011 with a standard deviation of 0.015 and an highest value of 0.11 . The immigrants with the highest value of the average co-ethnic network size are those from Western Europe (0.033) followed by Turkish immigrants (0.027), and South-Eastern European immigrants (0.019).

\subsection{Descriptive Statistics}

Table 1 reports summary statistics for the main variables used in our empirical analysis. The top panel of this table reports averages for time-varying individual variables. The first of them, however, Netw ${ }_{c d_{0}}$ measures the size of the co-ethnic network at time of arrival (described above) and is fixed for an individual. The employment probability has an average of 68.8 percent in the individual-year observations. ${ }^{27}$ The average wage per hour earned in the sample is around 8.5 Euros for full-time workers. Individuals in the sample are investing in education, i.e. spending some time in school or training, in 4.4 percent of the individual-year observations. Education and training are more likely when an immigrant first arrives and the share of individualyear in education is higher during the early years of their stay in Germany: twelve percent among immigrants in Germany for two years or less was in school part of the year, but that percentage was less than 2 for immigrants in Germany for at least six years (see Table D.2). Symmetrically, employment rates increase with time since arrival. During the first two years only 48 percent of individuals work, while after 10 years more than 76 percent are employed (see Table D.2). Our panel is unbalanced, the average number of years since migration observed is 7.56 , whereas the median value is 6 years. Around 23 percent of observations are relative to individuals who lived between zero and two years in Germany, 21 percent for three to five years, and 56 percent have been in Germany six or more years. Our sample is comprised of relatively young individuals - the average age is 37 years old.

The bottom panel of Table 1 lists averages of time-invariant individual characteristics mainly relative to ethnicity, country of origin and pre-migration characteristics. These information are obtained from the IAB-SOEP immigrant survey. Our sample consists of 933 foreign born individuals ${ }^{28}$ in working age (15-65 years old), who are

\footnotetext{
${ }^{27}$ We define employment as working for any extent of time during the year. This share falls to 56.3 percent if we count as employed only those working for at least 50 percent of days.

${ }^{28}$ Using country of birth to identify immigrants is a much more precise definition, and this represents an improvement with respect to all previous papers using German administrative data, which can only identify immigrants via nationality. This is particularly important for Germany, where the large group of ethnic Germans is entitled to receive the German nationality by law. To
} 
linked to the registry data. Among those immigrants, we select individuals whose date of arrival reported in the survey is within three years of their first appearance in the registry data. As we do not have information on the district of arrival from the survey, we take the district of first registration in the administrative data as capturing the place of arrival of the new immigrant. ${ }^{29}$ In addition to the standard characteristics, such as gender, age, and region of origin, we include a set of pre-migration characteristics that we use throughout the analysis: education, work experience, language proficiency, and employment status one year before migration. The survey data also report the job search method for the first job found in Germany, as well as a measure of self-assessed over-qualification in the current job. Our sample reflects the fact that people are relatively young when they migrate: age at migration is 30.5 years on average with a median age of 29 . We find that 54.3 percent of the immigrant sample found their first job in Germany through personal contacts, ${ }^{30} 63.5$ percent among low-skilled immigrants (those with at most lower secondary school). The information on job search method is rarely available in data that collect labor market outcomes. It allows us to test the importance of local co-ethnic networks in finding a job via personal referrals. Finally, 41.3 percent of those currently working report to have a degree higher than the level of schooling required for that job, which may suggest a certain degree of "downgrading" whereby individuals are matched to jobs that do not require the skills they have. ${ }^{31}$

the best of our knowledge, Dustmann et al. (2016) is the only other study using this definition of immigrant with German administrative data.

${ }^{29}$ Our results are robust to restricting the analysis to individuals whose year of arrival corresponds exactly to the first year in the registry data (55 percent). There are also cases in which the individual appears in the administrative data before the last migration year reported in the survey. In those cases we consider as year of arrival the first appearance in the registry data if the person appears working at least once in the subsequent years.

${ }^{30}$ The question asked is the following: "How did you find the first job in Germany?". The possible answers are: Federal Employment Office, employment agency, employment agency for foreigners, private job agency, job advertisement in the newspaper, job advertisement on the internet, through business relationships in Germany, through friends/acquaintances/relatives (which we denote as 'personal contacts'). We exclude here a small group of individuals who never work in the administrative archive or whose first job appears after 5 years since migration.

${ }^{31}$ Individuals who never work in the administrative data (4 percent) are not considered, since they may be working as self-employed. 


\section{Empirical Specification and Identification}

In order to estimate the effect of the size of the co-ethnic network at arrival on the employment and human capital investment of new immigrants, we adopt the following linear estimating equation:

$$
Y_{i c d_{0} t}=\alpha+\beta \mathbf{X}_{\mathbf{i t}}+\gamma_{0} N e t w_{c d_{0}}+\gamma_{1} N e t w_{c d_{0}} \times Y s m_{i t}+\eta Y s m_{i t}+\delta_{d_{0}}+\psi_{t_{0}}+\theta_{c}+\epsilon_{i t}
$$

Where $Y_{i c d_{0} t}$ is an outcome for individual $i$ from country-group of origin $c$ in year $t$ who first arrived in district $d_{0}$. In our main regressions the variable $Y$ will be, alternatively, a dummy for being employed or a dummy for being in school or training. $\mathbf{X}_{\mathbf{i t}}$ is a vector of time-varying and time-invariant individual characteristics and includes a gender dummy, age, age squared, age at migration and its square, and a set of pre-migration characteristics (education, working experience, employment, and language proficiency). The variable $N e t w_{c d_{0}}$ captures the size of the co-ethnic network (previous working immigrants from the same country-group $c$ as share of total employment) in the district of arrival $d_{0}$. It varies across country-groups and districts, and for each individual is fixed to the value in year 0 , the year of arrival. The term $\delta_{d_{0}}$ captures a set of district-of-arrival fixed effects and $\theta_{c}$ captures a set of country-of-origin fixed effects. The term $\psi_{t_{0}}$ is a year-of-arrival fixed effects. The variable $Y s m_{i t}$ is a dummy that indicates the number of years since migration for individual $i$. In the main analysis we use three dummies for "years since migration": $(Y s m 0-2)_{i t},(Y s m 3-5)_{i t}$ and $(Y s m 6+)_{i t}$, denoting the first two years, years 3-5, and more than five years from arrival, respectively. We also experiment with a more flexible specification including year dummies.

The nonrandom initial location of immigrants may bias the estimates of the coefficients of interest $\left(\gamma^{\prime} s\right)$ if unobserved individual characteristics affecting employment and human capital investments are also correlated with the initial size of the coethnic network. Controlling for pre-migration characteristics (usually not observed, but available in our data) and including district and country-of-origin fixed effects, which absorb systematic differences in economic performance across cities and ethnic groups, alleviates this issue substantially. In our main specification, we estimate equation (5) using OLS while absorbing location specific effects and pre-migration characteristics among the controls. We therefore only exploit differences in the size of initial co-ethnic network unrelated to pre-migration characteristics. We only use within-district, within country-of-origin variation, controlling for time-of-arrival ef- 
fects. Local district economic shocks that affect economic outcomes in subsequent years and the likelihood an immigrant moves there are absorbed by district-time effects. Similarly, specific characteristics linked to country of origin are absorbed by fixed country effects. We estimate equation (5) using the sample of all immigrants and a restricted sample whose members were initially dispersed and hence not subject to initial self-sorting. The restricted sample consists of people who reported in the survey entering Germany as asylum seekers or refugees ${ }^{32}$ and of ethnic Germans during the period of the Residence Allocation Act. Due to institutional arrangements, both of these groups were subject to a dispersal policy implemented by a central authority (see Appendix B for details about the Institutional setting). Ethnic Germans (Aussiedler) are migrants with German ethnicity mainly from Eastern Europe who were subject to a random allocation as they entered Germany. This restricted sample of refugees and ethnic Germans consists of 297 individuals, of whom about one-third are asylum seekers and two thirds ethnic Germans.

The comparison between the estimates in the overall sample and those in the restricted sample indicates whether a possible bias - induced by immigrant sorting still exists in the sample that decided their own location after controlling for a very rich set of covariates: district effects, country-of-origin effects and pre-migration individual characteristics. If most of the omitted variables bias is eliminated by this strategy, the full sample estimation should produce coefficients similar to those using the restricted sample. This would be a sign that the identified coefficient is consistent with causal interpretation. Considering the whole sample of immigrants, we also test for the individual determinants of sorting. Namely, we analyze whether the pre-migration individual characteristics - included as controls in the main analysis - are correlated with co-ethnic network size, and how this correlation is reduced when we include different sets of fixed effects. Both checks reassure us that omitted variable and selection concerns are effectively addressed by our method. In the most demanding specifications we control for a full battery of two-way fixed effects: country of origin by year of arrival, year of arrival by district of arrival, and country of origin by district of arrival fixed effects. ${ }^{33}$

\footnotetext{
${ }^{32}$ In the following we will denote this group as refugees for simplicity.

${ }^{33}$ In order to do this we need to rely on pre-estimated fixed effects computed using an external sample of immigrants obtained from administrative data, as our small sample size does not allow us to estimate them reliably.
} 


\section{Results}

\subsection{Employment}

Our main empirical results are illustrated in Tables 2 and 3. All of the tables described in this section use the same notation. The estimates in the row $\mathrm{Netw}_{c d_{0}}$ contain the coefficient on the size of the co-ethnic network in the district of arrival. The network variables we use throughout the analysis are standardized, i.e. they have mean zero and standard deviation one. Our estimates measure the impact of an increase in the size of co-ethnic network by one standard deviation on the outcome in the initial years (0-2) after arrival. The terms (Netw ${ }_{c d_{0}} \mathrm{xYsm3-5)}$ and $\left(\mathrm{Netw}_{c d_{0}} \mathrm{xYsm6}+\right)$ show the coefficients on the interactions of the initial network size with a dummy that is equal to one when individual $i$ has been in Germany between three and five years or more than five years, respectively.

The dynamic effects of the initial co-ethnic network on employment are estimated using a linear probability model, with a dummy for being employed in each year as our dependent variable. Results are reported in Table 2. In Column 1 we only include the non-interacted network size measure. This column is most similar to the type of "static" estimates previously presented in the literature (see Edin et al., 2003 and Damm, 2009 for a similar analysis on earnings.). On average, a larger co-ethnic network at arrival significantly increases the probability of employment. In Columns 2 and 3 we include the interactions with year since arrival and control for basic demographics and pre-migration characteristics, and include district, year and country-of-origin fixed effects. In Column 4 we control for the average wage in the district as an indicator of local productivity. In Column 5 we restrict the estimates to the sample of refugees and ethnic Germans (denoted as sample R), which approximates more closely the condition of initial random distribution of immigrants across districts. Columns 6 and 7 show our estimates where fixed effects (district, year and country of origin) are estimated on a large external sample of immigrants taken from administrative data. Columns 8 and 9 include external estimates of two-way fixed effects (country by district, district by year, country by year). The estimates are consistent with the basic prediction of our model, and their magnitude is very stable across specifications. Social networks have significantly positive effects on the probability of being employed and this effect is significant in the first two years after arrival. When we do not include dummies for years since arrival, we obtain a positive estimate on the network size that implies an increase of the probability 
of working by 4 percentage points (relative to an average employment rate of 68.8 percent) for an increase in the network by one standard deviation.

From Column 2 onwards, we estimate the effect interacted with year since arrival. For the first two years such an increase in probability of employment is much larger (about 10.7 percentage points), but the effect vanishes after around five years. The results also hold when we include the full set of pre-migration characteristics and fixed effects (Column 3) and when we include the average wage by district-year (Column 4). These controls should capture individual characteristics affecting employment probability and local labor demand conditions. Both could be correlated with the outcomes and with the sorting into initial networks of different size. ${ }^{34}$ The results hold when we perform the analysis on the restricted sample of asylum seekers and ethnic Germans (Column 5). Due to the drastic reduction in the sample size, the effect is less precisely estimated. One reason may be that the fixed effects we include are too numerous to be estimated with precision in the small sample of surveyed immigrants. Therefore, we replicate the analysis on both samples using pre-estimated country, year-of-arrival and district-of-arrival fixed effects (Columns 6 and 7). ${ }^{35}$ In addition, we use all possible two-way fixed effects (country of origin by year of arrival, year of arrival by district of arrival, and district of arrival by country of origin), also estimated out of sample, in Columns 8 and 9 , representing the most demanding specifications. It is reassuring to see the coefficients on all the network and interaction variables are quite similar to those estimated in Columns 4 and 5. The results remain significant after adopting different levels of clustering. In each table we report standard errors when clustering at the individual level (in parenthesis) as well as the more conservative clustering at the district level (in square brackets).

\footnotetext{
${ }^{34} \mathrm{An}$ even better control for labor demand factors would be the unemployment rate by district and year. Due to administrative changes in the registry data, we don't have this information for the aggregate data at district level for the years before 1999.

${ }^{35}$ The external estimation sample is a sample of 176,387 randomly drawn individuals with nonGerman nationality from the 2 percent IEB registry, corresponding to 1,569,520 person-year observations. The estimated regression includes gender, education, age, age squared and the fixed effects. Given the very high number of missing information for the education variable, the latter is imputed using the algorithm IP1 developed by Fitzenberger et al. (2005). We estimate these fixed effects and then import them into the main sample as additional regressors. For all regressions where we use predicted fixed effects, we obtain the standard errors using 500 bootstrap replications.
} 


\subsection{Human Capital Investments}

Differences in employment rates associated with large initial networks disappear over time. What offsetting factors were at work for individuals arriving in places with smaller co-ethnic networks? The survey includes information on the full history of human capital investments in Germany. We analyze whether there is a systematic relationship between social networks at arrival and investment in human capital. The main results of this regressions are presented in Table 3. There we find relatively strong evidence of a negative association between the size of the initial network and the probability of investing in human capital in the first five years. Immigrants arriving in cities with larger co-ethnic network by one standard deviation are 2.9 percentage points less likely to invest in human capital during their first two years (Column 2), where the baseline average is around twelve percent. The respective "static" effect is still negative and significant, however much lower in magnitude, corresponding to a reduction by one percentage point (Column 1). ${ }^{36}$ These results are consistent with our model, which predicts that individuals with larger initial co-ethnic networks are more likely to work and less likely to pursue more education in Germany. Results are robust to the inclusion of controls for pre-migration characteristics (Column 3), as well as to the inclusion of average wage at the district-year level (Column 4). In addition, they are robust and even stronger when we replicate the analysis for the restricted sample of refugees and ethnic Germans (Column 5), corresponding to a 7.5 percentage points reduction in the probability of investing in human capital. ${ }^{37}$ The results on employment and human capital investment are robust to restricting the sample to individuals who were younger than 24 or 29 at migration.

\subsection{Effects by Education Group}

We then analyze heterogeneity according to initial human capital. Table 4 (Columns 1-3) breaks down the main sample by pre-migration educational attainments. ${ }^{38}$ The

\footnotetext{
${ }^{36}$ To the best of our knowledge this is the first estimate in the literature of the effect of co-ethnic networks at arrival on human capital investment of immigrants.

${ }^{37}$ We cannot add pre-estimated two-way fixed effects as in the employment regressions because we lack a comparable sample with information on human capital investments.

${ }^{38}$ In all our analysis, the breakdown by education is always obtained considering the education obtained abroad by the year of arrival in Germany. Three education categories are considered following the standard German classification: low skill corresponding to no vocational training, medium skill corresponding to some form of post-secondary vocational study, and high skill corresponding
} 
first three columns estimate the effect of the network and its interactions with years since migration on employment probability, separately by education group. The overall positive initial effect of network on employment is stronger for low- and medium-skilled immigrants, while it is close to zero and not statistically significant for highly educated immigrants. We also find, as in Table 2, that the effect disappears around six years after arrival. Figure 3 plots the estimated coefficients of the yearly interactions, together with the 90 and $95 \%$ confidence intervals, by year since arrival. The top-left panel shows estimates on the full sample and the other three panels for the education groups separately. The coefficient is significantly positive in the early years and it converges to zero after about $4-5$ years. For less-educated immigrants the effect of co-ethnic network in job finding is substantial. Moving to a district with one standard deviation larger co-ethnic network at arrival corresponds to a 15 percentage points greater probability of being employed. Also, for the less-educated immigrants this effect disappears four years after migration. The network effects is still positive, though significantly lower ( 8 percentage points for each one-standarddeviation increase) for immigrants with intermediate levels of schooling. It is not significantly different from zero for the highly skilled.

Columns 4-6 of Table 4 investigate the relationship between network size and human capital investment for individuals with different initial education levels. Results are again driven by low- and medium-skilled workers, which is consistent with the predictions of our model. There are no significant effects for highly-educated workers. Our findings suggest that, after arrival, less educated immigrant workers in places with large co-ethnic networks find employment with larger probability. The benefits of networks, however, dissipate over time. This may be because individuals in location with smaller networks invest more in human capital and in the long run have the same probability of being employed as the group that started with a larger co-ethnic network. Short-run effects on employment probability and human capital investment are larger for the low and medium educated. In the first two years after arrival they experience lower probability of going back to school by 3.2-3.1 percentage points if they land in a district with one standard deviation larger co-ethnic networks. This difference, however, disappears after 4-5 years. In the sample of high skilled, the effects are close to zero in magnitude and not statistically significant. For the yearly effects see Figure 4.

to college education and above. 


\subsection{Wage Effects}

Table 5 analyzes the impact of co-ethnic networks on wages. In the first column we present the coefficients estimated on the full (1) and restricted (2) samples. Columns 3-5 show the results separating individuals with low, medium and high education. When we consider the full sample of immigrant workers (Column 1) we do not detect significant effects on wages in the short run. In the long run, an insignificant negative effect is present. We find, however, a negative effect on the restricted sample in the short term (Column 2), which is substantial in magnitude (20.7 percent, or a log difference of 0.233 ), and significant at the $1 \%$ level. The negative effect for this sample decreases but persists also in the long term (18.2 percent, or a log difference of 0.202 ) and remains statistically significant. Once we break down the sample by skill group (Columns 3-5), the evidence suggests a negative and significant effect of initial network only for the low skilled. Differently from the effect on employment, the effect on wages for less educated persists beyond the short-term and remains statistically significant: in the first two years since migration the reduction in hourly wages for each standard-deviation increase in network size corresponds to 7.5 percent (a $\log$ difference of 0.079), and it increases to 9.4 percent after six or more years. ${ }^{39}$ Such an effect is consistent with the hypothesis that initial network-generated job referrals imply somewhat lower quality jobs. Once in these jobs, individuals miss on human capital accumulation and their wage progression is not as fast as elsewhere, hence they maintain this initial disadvantage.

According to the predictions of our simple theoretical setup, without additional assumptions on the wage distribution of the two channels there is not clear prediction on initial relative observed wages. Assuming that the two distributions have the same expected value, there is a positive relationship between network and observed initial wages. This prediction, however, seems not to be supported by our empirical results for the low skilled. One possible explanation, also developed theoretically by Bentolila et al. (2010), is that there is matching specific heterogeneity in productivity across formal and informal channel. In particular, finding a job through the informal

\footnotetext{
${ }^{39}$ The literature provides mixed evidence about the effect of social networks on wages also for the general population. In the seminal work of Granovetter (1973) the quality of the match depends on how close the person is to the social contact. More recently, Loury (2006) finds that the wage effect varies according to the type of contacts, whereas Pellizzari (2010) shows the positive effect on wages of finding the job through the informal channel rises when the efficiency of the matching process in the formal channel increases as firms become more selective when hiring through the informal channel. Looking at the effect of co-ethnic networks on earnings, Damm (2009) finds a substantial positive effect, equivalent to 18 percent, irrespective of the skill level.
} 
channel may be associated with a penalty due to imperfect matching. ${ }^{40}$ Indeed, if one postulated wage offers through the informal channel to be on average lower than those of the formal channels, this is the result that one would get. We also implement a more flexible specification, including yearly dummies interacted with initial network size. The estimates as well as the $90 \%$ and $95 \%$ confidence intervals for the coefficients on year since migration dummy are reported graphically in Figure 5. The effect on wages is null for the full sample (top left panel) as well as for the medium and high skilled, whereas it is negative and stable in the long run for the low skilled (top right panel), with a magnitude of around 10 percent.

In the model developed above, jobs found through networks and those found in the formal channel were not different in their quality or type. In a more general setup, one might expect that ethnic networks of immigrants may be particularly good in generating referrals for jobs that do not require a high level of formal education and are in specific labor market niches. These jobs may be more easily signaled to co-nationals of existing workers, but they may also be an imperfect match for the specific abilities of a new immigrant and have low potential of generating a professional career, similarly to Bentolila et al. (2010). We investigate this mechanism by analyzing whether the larger probability of employment associated with a larger size of co-ethnic networks is also accompanied by a larger "mismatch" on the job. We construct a measure of job mismatch from the information in the survey. Individuals are asked about the type of education required for the current occupation and this information is compared to the education that they effectively have. Individuals are classified as overqualified when the education level required is lower than their level of education; we use a dummy to indicate whether individuals are overqualified. Given that this information is only available for the last job, we estimate the same specification (5) as in the rest of the analysis, but using only one observation for each individual and, hence, we simply estimate the effect of network at arrival on current job mismatch. Moreover, we pool all individuals and include a "low-education dummy" and its interaction with the size of the initial network.

The first row of Table 6 shows the coefficient on the low-education dummy, the second row the coefficient on the initial co-ethnic network and the third row is the additional effect of the network on less educated. Specifications from (1) to (4) add progressively more controls. The estimated coefficients in Table 6 should be inter-

\footnotetext{
${ }^{40}$ In our data, the first job found through the network is unconditionally associated with a 13 percent lower wage than jobs found through other methods.
} 
preted as the impact of initial co-ethnic network size on the probability of being over-qualified in the current job. We find statistically significant and economically large effects of network size for less skilled. Workers who migrated with less education are more likely to be - years later - in a job for which they are overqualified when arriving in districts with larger co-ethnic networks. For low-skilled individuals, the increase in the initial network size by one standard deviation corresponds to an 10-percentage-point-higher likelihood of being overqualified for the current job. This magnitude translates into 24 percent of the average (41.3 percent, standard specification in Column 1) and is robust to controlling for pre-migration characteristics (Column 2), as well as to the average wage at district-year of arrival (Column 3), or past cognitive skills (Column 4), measured as the self-reported past test score in math. There is no significant effect of initial network size for the medium skilled, nor for the high skilled - our excluded category.

\subsection{Networks and Job Search Methods}

We have interpreted the higher probability of early employment for immigrants arriving in locations with large networks as a result of higher probability of being refereed to a job by such network. This channel, however, has not been directly tested in the literature. ${ }^{41}$ The reason is that, in most datasets, it is not possible to know whether and how search methods are affected by the size of social networks. In our data, we have direct information on the way an individual has found her first job in Germany. This unique information allows us to investigate the effect of co-ethnic networks at arrival on the type of search method used to find the first job in Germany. Table 7 uses - as the dependent variable - a dummy equal to one if the first job in Germany was found thanks to "personal contacts". Personal contacts - according to the questionnaire - refer to friends, relatives, or acquaintances. We estimate a similar specification as in Table 6 , in that we use the cross-section of immigrants, we control for their initial characteristics, and we estimate the impact of initial network size on the probability of having found the first job through personal contacts.

The results show a significant positive association between initial network size and the likelihood that the first job in Germany was found through personal contacts, and

\footnotetext{
${ }^{41}$ Dustmann et al. (2016) is a notable exception. The authors use German administrative data to evaluate the effect of within-firm ethnic networks on wage growth and firm turnover. They use the same survey that we use to show how the within-firm ethnic networks affect the probability of finding the job through contacts.
} 
this is shown in Column 1 of Table 7. In particular, one standard deviation increase in the co-ethnic network size at arrival corresponds to a 5.5 percentage points greater likelihood of having found the first job through contacts. Columns 2-4 show that this overall effect is driven by immigrants with moderate levels of education, for whom the equivalent effect is larger at around 9 percentage points. This magnitude corresponds to around 16 percent of the average (which is 54.3 percent) and is robust to the inclusion of the usual controls and the average wage at the district of arrival. Table D.3 in the Appendix presents the results from similar regressions (the main results of Table 7, Column 3, are presented in Column 1 for comparison) where the dependent variables are dummies equal to one if an individual found a job using online advertising (Column 2) or if she used an employment agency (Column 3). The increased reliance on personal contacts by individuals with lower levels of education arriving in districts with larger networks corresponds to a decreased reliance on Internet and employment agencies. ${ }^{42}$

\subsection{Falsification Exercises and Robustness Checks}

In our main specification, we calculate network size as the share of employed individuals from the own country group in the district of arrival. A possible concern with our findings is that they may be driven by strong labor demand conditions for immigrants in a district. In this case, our network measure based on individuals from the same origin country would in part measure these favorable conditions. Hence, in Table 8 we perform a placebo-type analysis where we investigate the specific role of co-ethnic network, as opposed to that of the generic share of immigrants. In Columns 2 and 4 of Table 8 , we use as explanatory variable $\left(\operatorname{Netw}_{d} 0\right)$, the number of all immigrants excluding co-nationals, as share of the employment of a district. Columns 1 and 2 show the estimates of the network variable and its interactions for the employment regressions: Column 1 reports the baseline estimates and Column 2 uses the share of foreign born excluding the co-nationals in the same district. The estimates of Column 2 are not significantly different from zero, and are of very small magnitude. This finding is consistent with our view that co-ethnic networks are the determinants of the employment effect, and the results presented are not simply an

\footnotetext{
${ }^{42}$ It is worth noting here that the results of these regressions do not refer to the relative time spent searching. Rather, they concern only the successful search method, because our question on job search methods refers to the first job found in Germany. Therefore, we do not know whether and to what extent other search methods have been used.
} 
effect of local labor market demands (proxied by a general measure of immigration).

Columns 3 and 4 perform an equivalent falsification test on the relationship between network size and human capital investment. Column 3 presents our original results. In Column 4 we define networks as the share of non-co-national foreign born in local employment. Effects are not significantly different from zero, and very small in magnitude. The results of this exercise are consistent with the mechanism operating at the level of the co-ethnic network. In Appendix D, we perform an additional robustness check adding a measure of cognitive skills (self-reported past test score in math, see Table D.4) to our regression. ${ }^{43}$ Our results on employment (Columns 1 and 2) and on human capital (Columns 3 and 4) are confirmed.

\subsection{Initial Location Decision}

Our survey includes a rich set of information on pre-migration characteristics: education, employment, working experience, language proficiency, cognitive ability. In this section, rather than simply using those characteristics as controls, we use them to test the initial sorting of immigrants across locations, which provides an idea of the potential endogeneity concerns for our estimates. In particular, while initial characteristics of immigrants can be correlated with the size of co-ethnic networks at arrival because different people have different preferences for networks, we test whether this correlation survives the inclusion of district, country-of-origin, and year fixed effects. If the correlation between pre-migration characteristics and size of the network is weak once we condition on our set of fixed effects, this would imply that individual characteristics do not explain the initial location, and hence, selection, at least on observables, is not too concerning. If the vector of pre-migration observable characteristics is a good proxy for unobserved characteristics affecting wage and employment after migration, this test is a check of how severe the omitted variable bias can be in our regressions. This test of orthogonality is novel in this literature. It is generally not possible to implement using social security data alone because information about pre-migration variables is usually unavailable. Testing the conditional orthogonality of the size of the co-ethnic network and individual characteristics is not a perfect test of randomness, but it provides an important check on the correlation between network size and potentially omitted variables that may affect labor market outcomes. A similar exercise is carried out in Guryan et al. (2009) to test

\footnotetext{
${ }^{43}$ We are not including this measure in our main regressions because we would lose around 29 percent of our sample (the relative question is asked only in the second wave of the survey).
} 
the orthogonality between predetermined student characteristics and average class characteristics. The exercise consists of regressing the initial network size variable on all pre-migration variables (Table 9), first without other controls (Column 1), then adding country-of-origin, arrival year, and arrival district fixed effects (Columns 2 and 3). While significant correlations exist (showing in particular that large initial co-ethnic networks are associated with less educated immigrants and workers with less work experience), none of the pre-migration variables is correlated with the initial network size (neither individually nor jointly) once we add our controls. once we condition on district, year of arrival, and country fixed effects, there is no remaining correlation between any pre-migration characteristic and the size of the initial network. Given this evidence, we can argue, once we include a full set of fixed effect dummies, our results are not significantly affected by sorting.

Finally, we check that our results are robust to different geographical levels at which one can measure co-ethnic networks. Throughout the paper we use districts as units. Here, instead, we use municipalities, i.e. smaller units, of which there are about 12,000 in Germany. Table D.6 in Appendix D shows that our main estimates are robust to this change. ${ }^{44}$

\section{Concluding Remarks}

This paper looks at co-ethnic networks of immigrants in Germany and their role in helping the economic success of new immigrants. In particular, we investigate how the size of co-ethnic networks at arrival affects employment and human capital investments of immigrants soon after arrival, and in the following years. We develop a simple search model where individuals can search through a formal channel and an informal, network-based channel. In the informal channel, co-ethnic networks help individuals find employment by providing referrals. Such a model predicts an initial lower probability of employment for individuals with smaller size of coethnic networks. Over time, our model predicts convergence to similar employment probability and possibly higher wages for those with smaller co-ethnic networks because of higher human capital investments.

Our main dataset combines a novel survey of immigrants to Germany with administrative records to reconstruct the entire individual labor market history of recent

\footnotetext{
${ }^{44}$ Other papers also use small units, such as Bayer et al. (2008) who use Census blocks, whereas Schmutte (2015) considers small neighborhoods.
} 
immigrants, as well as their district of arrival in Germany. Our empirical evidence is consistent with the main implications of our model: individuals with larger ethnic networks are more likely to be employed soon after arrival, but are less likely to invest in human capital and are no more likely to be employed several years after arrival. In addition, the initial co-ethnic network exerts a negative long run effect on wages and on match quality. These effects are stronger for initially less-educated immigrants.

Identifying the effect of co-ethnic networks on human capital investment of recent immigrants is a new contribution of this paper, and suggests that, while positive overall, co-ethnic networks may give a larger initial boost that attenuates over time and can cause under-investment in human capital. Previous empirical estimations of network effects for immigrants such as Edin et al. (2003) and Damm (2009) emphasized only the earnings effect and lacked a dynamic analysis. As they found a positive impact of networks on earnings, they argued that dispersal policies have high costs for immigrants, worsening their labor market outcomes. The implications from our results, however, suggest a more complex story. While in the short-run employment probability may be increased by the presence of co-ethnic networks, dynamically they may reduce human capital accumulation and lower the quality of job matches and wages. Ignoring those effects may result in overestimating the negative effects of dispersal policies. Thanks to better data (inclusive of pre-migration characteristics) we can contribute to the literature by identifying with greater confidence the causal effect of co-ethnic networks on several different outcomes of immigrants in the short and in the long run. 


\section{Tables and Figures}

Table 1: Summary Statistics

\begin{tabular}{|c|c|c|c|}
\hline Variable & Mean & Std. Dev. & $\mathrm{N}$ \\
\hline & \multicolumn{3}{|c|}{ Time Variant Variables } \\
\hline Netw $_{c d_{0}}$ & 0.012 & 0.018 & 12241 \\
\hline Employment & 0.688 & 0.463 & 12241 \\
\hline Human Capital Investment & 0.044 & 0.205 & 12186 \\
\hline Real Hourly Wage & 8.506 & 4.003 & 4821 \\
\hline Year since Migr:0-2 & 0.228 & 0.419 & 12241 \\
\hline Year since Migr:3-5 & 0.214 & 0.410 & 12241 \\
\hline Year since Migr:6+ & 0.558 & 0.497 & 12241 \\
\hline \multirow[t]{2}{*}{ Age } & 37.017 & 10.452 & 12241 \\
\hline & \multicolumn{3}{|c|}{ Individual Variables } \\
\hline West & 0.105 & 0.307 & 933 \\
\hline East Eu & 0.129 & 0.335 & 933 \\
\hline Turkey & 0.064 & 0.245 & 933 \\
\hline South and East EU & 0.202 & 0.401 & 933 \\
\hline USSR & 0.427 & 0.495 & 933 \\
\hline Asia & 0.047 & 0.212 & 933 \\
\hline Africa & 0.020 & 0.141 & 933 \\
\hline Central and South America & 0.006 & 0.080 & 933 \\
\hline First Job Found through Contacts & 0.543 & 0.498 & 806 \\
\hline Low Edu & 0.421 & 0.494 & 933 \\
\hline Pre Migration Edu: Medium & 0.339 & 0.474 & 933 \\
\hline Pre Migration Edu: High & 0.240 & 0.427 & 933 \\
\hline Pre migration Employment & 0.726 & 0.446 & 929 \\
\hline Pre migration Language Proficiency & 0.115 & 0.319 & 930 \\
\hline Pre migration work Experience & 10.245 & 9.716 & 933 \\
\hline Age at Migration & 30.504 & 9.895 & 933 \\
\hline Overqualified in current Job & 0.413 & 0.493 & 644 \\
\hline
\end{tabular}

Source: IAB-SOEP Migration Sample linked to IEB. 


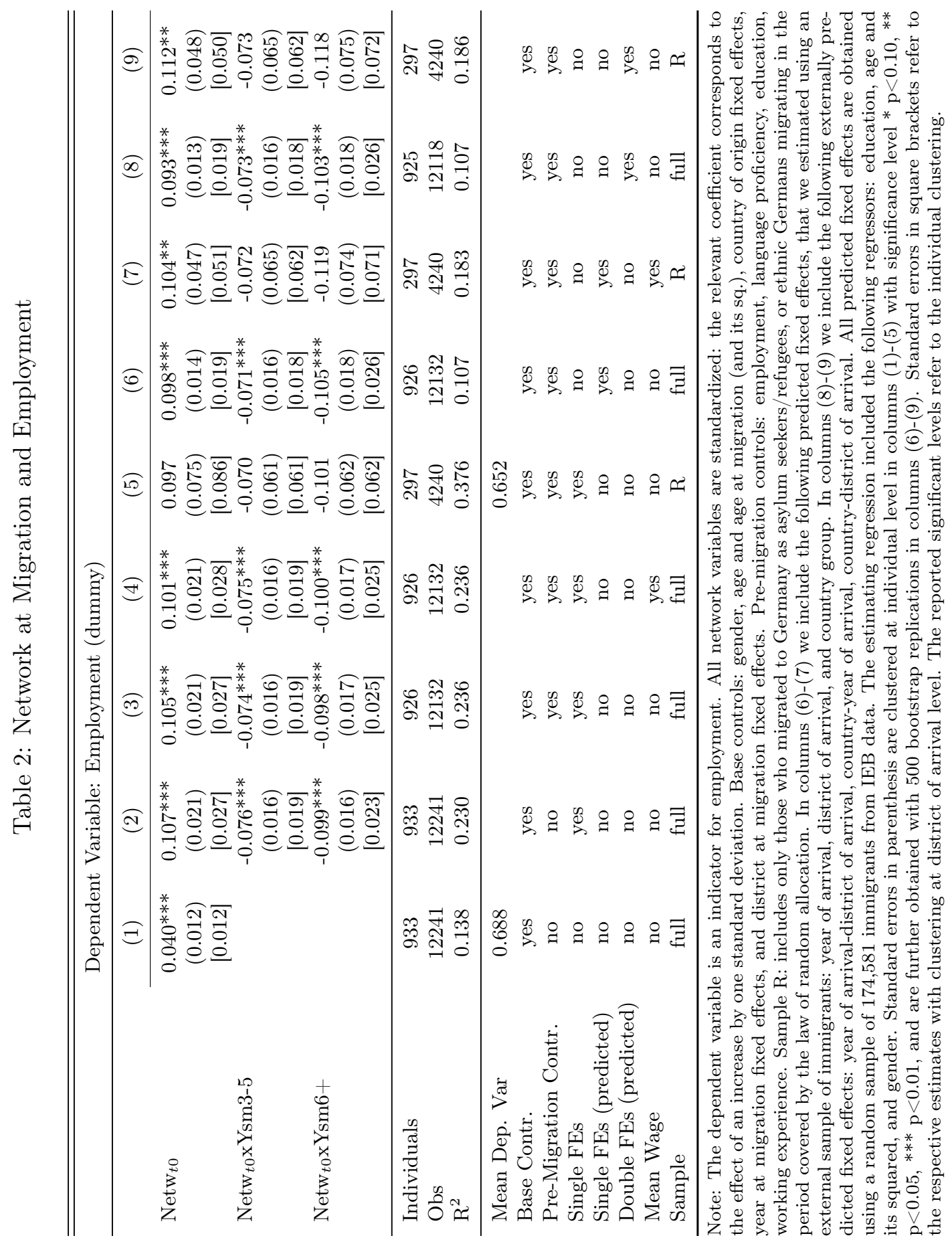


Table 3: Network at Migration and Investment in Human Capital

\begin{tabular}{|c|c|c|c|c|c|}
\hline \multicolumn{6}{|c|}{ Dependent Variable: Investment in Human Capital (dummy) } \\
\hline \multirow[b]{2}{*}{ Netw $_{t 0}$} & $(1)$ & $(2)$ & $(3)$ & $(4)$ & $(5)$ \\
\hline & $\begin{array}{c}-0.008^{* *} \\
(0.003) \\
{[0.003}\end{array}$ & $\begin{array}{c}-0.029 * * * \\
(0.009) \\
{[0.009]}\end{array}$ & $\begin{array}{c}-0.028^{* * *} \\
(0.009) \\
{[0.009]}\end{array}$ & $\begin{array}{c}-0.028^{* * *} \\
(0.009) \\
{[0.010]}\end{array}$ & $\begin{array}{c}-0.075^{* * *} \\
(0.026) \\
{[0.027]}\end{array}$ \\
\hline Netw $_{t 0} \times Y_{s m} 3-5$ & & 0.012 & 0.011 & 0.011 & 0.033 \\
\hline \multirow{5}{*}{ Netw $_{t 0} \times Y s m 3-5$} & & $(0.009)$ & $(0.009)$ & $(0.009)$ & $(0.020)$ \\
\hline & & {$[0.009]$} & {$[0.009]$} & {$[0.009]$} & {$[0.021]$} \\
\hline & & $0.031^{* * *}$ & $0.031^{* * *}$ & $0.031 * * *$ & $0.061^{* * *}$ \\
\hline & & $(0.009)$ & $(0.009)$ & $(0.009)$ & $(0.019)$ \\
\hline & & [0.010] & {$[0.010]$} & {$[0.010]$} & {$[0.021]$} \\
\hline Individuals & 933 & 933 & 926 & 926 & 297 \\
\hline Obs & 12186 & 12186 & 12094 & 12094 & 4233 \\
\hline $\mathrm{R}^{2}$ & 0.141 & 0.206 & 0.209 & 0.209 & 0.327 \\
\hline Mean Dep. Var & 0.043 & & & & 0.061 \\
\hline Pre-Migration Contr. & no & no & yes & yes & yes \\
\hline Mean Wage & no & no & no & yes & no \\
\hline Sample & full & full & full & full & $\mathrm{R}$ \\
\hline
\end{tabular}

Note: The dependent variable is an indicator for being in education. Network variables are standardized. Base controls (in all regressions): gender, age and age at migration (and its sq.), country of origin fixed effects, year at migration fixed effects, and district at migration fixed effects. Pre-migration controls: employment, language proficiency, education, working experience. Sample R: refugees and ethnic Germans. Standard errors in parenthesis are clustered at individual level, ${ }^{*} \mathrm{p}<0.10,{ }^{* *} \mathrm{p}<0.05,{ }^{* * *} \mathrm{p}<0.01$. We report standard errors clustered at district level in square brackets. 
Table 4: Network, Employment and Human Capital Investment by Education

\begin{tabular}{lcccccc}
\hline \hline \multirow{2}{*}{$\begin{array}{l}\text { Dep. Var. } \\
\text { Education }\end{array}$} & \multicolumn{3}{c}{ Work/Not Work } & & \multicolumn{3}{c}{ HC Investment } \\
& Low & Medium & High & Low & Medium & High \\
\hline \multirow{2}{*}{ Netw $_{t 0}$} & $(1)$ & $(2)$ & $(3)$ & $(4)$ & $(5)$ & $(6)$ \\
\cline { 2 - 7 } & $0.146^{* * *}$ & $0.079^{*}$ & 0.003 & $-0.032^{* *}$ & $-0.031^{* * *}$ & 0.005 \\
& $(0.027)$ & $(0.041)$ & $(0.059)$ & $(0.016)$ & $(0.009)$ & $(0.025)$ \\
Netw $_{t 0} \times$ Ysm3-5 & {$[0.033]$} & {$[0.041]$} & {$[0.073]$} & {$[0.017]$} & {$[0.010]$} & {$[0.023]$} \\
& $-0.079^{* * *}$ & $-0.097^{* * *}$ & -0.039 & 0.021 & $0.013^{*}$ & 0.008 \\
Netw $_{t 0} \times$ Ysm6+ & $(0.025)$ & $(0.033)$ & $(0.030)$ & $(0.014)$ & $(0.008)$ & $(0.019)$ \\
& {$[0.024]$} & {$[0.036]$} & {$[0.035]$} & {$[0.015]$} & {$[0.007]$} & {$[0.021]$} \\
& $-0.121^{* * *}$ & $-0.076^{* *}$ & -0.068 & $0.044^{* * *}$ & $0.026^{* * *}$ & 0.015 \\
& $(0.024)$ & $(0.030)$ & $(0.041)$ & $(0.015)$ & $(0.009)$ & $(0.019)$ \\
Individuals & {$[0.029]$} & {$[0.030]$} & {$[0.052]$} & {$[0.016]$} & {$[0.010]$} & {$[0.020]$} \\
$\mathrm{R}^{2}$ & 389 & 314 & 223 & 389 & 314 & 223 \\
\hline Mean Dep. Var & 5445 & 4031 & 2656 & 5424 & 4031 & 2639 \\
\hline
\end{tabular}

Note: The dependent variable is an indicator for employment (columns 1-3), or a indicator for being in education (columns 4-6). Education is measured before migration. Low education refers to lower secondary education. Medium and high refer to upper secondary, and tertiary education, respectively. All network variables are standardised. Controls include base controls and pre-migration controls. Base controls: gender, age and age at migration (and its sq.), country of origin fixed effects, year at migration fixed effects, and district at migration fixed effects. Pre-migration controls: employment, language proficiency, education, working experience. Standard errors in parenthesis are clustered at individual level, $* \mathrm{p}<0.10, * *$ $\mathrm{p}<0.05, * * * \mathrm{p}<0.01$. We report standard errors clustered at district level in square brackets.

Table 5: Network and Wages

\begin{tabular}{|c|c|c|c|c|c|}
\hline \multicolumn{6}{|c|}{ Dependent Variable: Hourly Wage (log) } \\
\hline \multirow[t]{2}{*}{ Education } & & & Low & Medium & High \\
\hline & $(1)$ & $(2)$ & $(3)$ & $(4)$ & $(5)$ \\
\hline $\operatorname{Netw}_{t 0}(\mathrm{a})$ & $\begin{array}{l}-0.007 \\
(0.032) \\
{[0.034]}\end{array}$ & $\begin{array}{c}-0.233^{* * *} \\
(0.086) \\
{[0.111]}\end{array}$ & $\begin{array}{c}-0.079^{* *} \\
(0.033) \\
{[0.036]}\end{array}$ & $\begin{array}{c}0.080 \\
(0.058) \\
{[0.070]}\end{array}$ & $\begin{array}{l}-0.127 \\
(0.154) \\
{[0.154]}\end{array}$ \\
\hline \multirow[t]{3}{*}{ Netw $t 0 \times$ Ysm3-5 } & -0.017 & $0.059^{*}$ & -0.010 & $-0.052^{*}$ & -0.010 \\
\hline & $(0.015)$ & $(0.032)$ & $(0.022)$ & $(0.029)$ & $(0.025)$ \\
\hline & [0.015] & {$[0.032]$} & {$[0.021]$} & {$[0.028]$} & {$[0.027]$} \\
\hline \multirow{3}{*}{ Netw $t 0_{t} \times Y_{s m 6}+(b)$} & -0.030 & 0.031 & -0.020 & -0.009 & $-0.076^{* *}$ \\
\hline & $(0.018)$ & $(0.041)$ & $(0.024)$ & $(0.034)$ & $(0.037)$ \\
\hline & {$[0.016]$} & {$[0.042]$} & {$[0.020]$} & {$[0.032]$} & {$[0.038]$} \\
\hline Individuals & 668 & 216 & 277 & 228 & 163 \\
\hline Obs & 4934 & 1650 & 2151 & 1723 & 1060 \\
\hline & 0.489 & 0.647 & 0.593 & 0.627 & 0.691 \\
\hline$(a)+(b)==0(p-$ value $)$ & 0.191 & 0.010 & 0.000 & 0.181 & 0.191 \\
\hline Mean Dep. Var & 8.498 & 7.656 & 7.702 & 7.782 & 11.368 \\
\hline Sample & full & $\mathrm{R}$ & full & full & full \\
\hline
\end{tabular}


Table 6: Network at Migration and Over-qualification in the current Job

\begin{tabular}{|c|c|c|c|c|}
\hline \multicolumn{5}{|c|}{ Dependent Variable: Over-qualification (dummy) } \\
\hline & $(1)$ & $(2)$ & $(3)$ & $(4)$ \\
\hline Low Edu & $\begin{array}{c}-0.240^{* *} \\
(0.112)\end{array}$ & $\begin{array}{c}-0.243^{* *} \\
(0.121)\end{array}$ & $\begin{array}{c}-0.240^{*} \\
(0.122)\end{array}$ & $\begin{array}{c}-0.282^{* *} \\
(0.138)\end{array}$ \\
\hline Netw $_{t 0}(\mathrm{a})$ & $\begin{array}{l}-0.012 \\
(0.051)\end{array}$ & $\begin{array}{l}-0.012 \\
(0.049)\end{array}$ & $\begin{array}{l}-0.016 \\
(0.048)\end{array}$ & $\begin{array}{l}-0.035 \\
(0.058)\end{array}$ \\
\hline Netw $_{t 0} \times$ Low Edu $t 0(\mathrm{~b})$ & $\begin{array}{c}0.113^{* *} \\
(0.047)\end{array}$ & $\begin{array}{c}0.110^{* *} \\
(0.048)\end{array}$ & $\begin{array}{c}0.111^{* *} \\
(0.048)\end{array}$ & $\begin{array}{c}0.122^{* *} \\
(0.055)\end{array}$ \\
\hline Individuals & 644 & 639 & 639 & 518 \\
\hline $\mathrm{R}^{2}$ & 0.617 & 0.624 & 0.625 & 0.647 \\
\hline$(\mathrm{a})+(\mathrm{b})==0(\mathrm{p}$-value $)$ & 0.023 & 0.027 & 0.029 & 0.078 \\
\hline Mean Dep. Var & 0.413 & & & \\
\hline Pre-Migration Controls & no & yes & yes & yes \\
\hline Average Wage at Dist $_{t 0} \times$ Year $_{t 0}$ & no & no & yes & no \\
\hline Past Cognitive Skill & no & no & no & yes \\
\hline
\end{tabular}

Table 7: Network and first Job in Germany found through Contacts

\begin{tabular}{|c|c|c|c|c|}
\hline \multicolumn{5}{|c|}{ Dependent Variable: Job Found through Contacts } \\
\hline & $(1)$ & $(2)$ & $(3)$ & $(4)$ \\
\hline Low $\mathrm{Edu}_{t 0}$ & & $\begin{array}{c}0.101^{*} \\
(0.060)\end{array}$ & $\begin{array}{c}0.090 \\
(0.062)\end{array}$ & $\begin{array}{c}0.090 \\
(0.062)\end{array}$ \\
\hline Netw $_{t 0}(\mathrm{a})$ & $\begin{array}{c}0.055^{* * *} * \\
(0.016)\end{array}$ & $\begin{array}{l}-0.006 \\
(0.055)\end{array}$ & $\begin{array}{l}-0.008 \\
(0.054)\end{array}$ & $\begin{array}{l}-0.003 \\
(0.055)\end{array}$ \\
\hline Netw $_{t 0}$ xLow Edu E $_{t 0}(\mathrm{~b})$ & & $\begin{array}{l}0.087^{*} \\
(0.045)\end{array}$ & $\begin{array}{c}0.098^{* *} \\
(0.045)\end{array}$ & $\begin{array}{c}0.099^{* *} \\
(0.044)\end{array}$ \\
\hline Individuals & 806 & 806 & 799 & 799 \\
\hline $\mathrm{R}^{2}$ & 0.013 & 0.419 & 0.428 & 0.429 \\
\hline$(a)+(b)==0(p-$ value $)$ & & 0.099 & 0.075 & 0.057 \\
\hline Mean Dep. Var. & 0.543 & & & \\
\hline Base Controls & no & yes & yes & yes \\
\hline Pre-Migration Controls & no & no & yes & yes \\
\hline Average Wage at Dist $t 0 \times$ Year $_{t 0}$ & no & no & no & yes \\
\hline
\end{tabular}


Table 8: Falsification Test: Network of all Other Immigrants

\begin{tabular}{lcccc}
\hline \hline Dependent Variable: & \multicolumn{2}{c}{ Employment } & \multicolumn{2}{c}{ Human Capital } \\
\hline Network: & Baseline & Other & Baseline & Other \\
\cline { 2 - 5 } & $(1)$ & $(2)$ & $(3)$ & $(4)$ \\
\hline Netw $t 0$ & $0.105^{* * *}$ & -0.007 & $-0.028^{* * *}$ & 0.002 \\
& $(0.021)$ & $(0.037)$ & $(0.009)$ & $(0.013)$ \\
Netw $t 0$ XYsm3-5 & $-0.074^{* * *}$ & -0.012 & 0.011 & 0.000 \\
& $(0.016)$ & $(0.014)$ & $(0.009)$ & $(0.007)$ \\
Netw $t 0$ XYsm6+ & $-0.098^{* * *}$ & -0.025 & $0.031^{* * *}$ & 0.000 \\
& $(0.017)$ & $(0.015)$ & $(0.009)$ & $(0.008)$ \\
\hline Individuals & 926 & 926 & 926 & 926 \\
Obs & 12132 & 12132 & 12094 & 12094 \\
$\mathrm{R}^{2}$ & 0.236 & 0.230 & 0.209 & 0.206 \\
\hline
\end{tabular}

Note: The dependent variable is an indicator for employment (columns 1-2), and an indicator for being in education (columns $3-4)$. In column (2) and (4) the network variable is computed using all immigrants in the district of arrival excluding those from the country of origin of the individual. Controls include base controls and pre-migration controls. Base controls: gender, age at migration (and its sq.), country of origin fixed effects, year at migration fixed effects, and district at migration fixed effects. Pre-migration controls: employment, language proficiency, education, working experience. All network Variables are standardised. Standard errors in parenthesis are clustered at individual level, ${ }^{*} \mathrm{p}<0.10,{ }^{* *} \mathrm{p}<0.05,{ }^{* * *} \mathrm{p}<0.01$.

Table 9: Test of Network Sorting

\begin{tabular}{|c|c|c|c|}
\hline \multicolumn{4}{|l|}{ Dependent Variable: Netw $_{c d_{0}}$} \\
\hline Pre-Migration Variable & $(1)$ & $(2)$ & $(3)$ \\
\hline Language & $\begin{array}{c}0.104 \\
(0.101) \\
{[0.120]}\end{array}$ & $\begin{array}{l}-0.035 \\
(0.071) \\
{[0.082]}\end{array}$ & $\begin{array}{l}-0.019 \\
(0.084) \\
{[0.101]}\end{array}$ \\
\hline Employment & $\begin{array}{l}-0.090 \\
(0.074) \\
{[0.071]}\end{array}$ & $\begin{array}{c}0.013 \\
(0.049) \\
{[0.050]}\end{array}$ & $\begin{array}{c}0.061 \\
(0.055) \\
{[0.065]}\end{array}$ \\
\hline Work Experience & $\begin{array}{c}-0.011^{* * * *} \\
(0.003) \\
{[0.003]}\end{array}$ & $\begin{array}{c}0.000 \\
(0.002) \\
{[0.002]}\end{array}$ & $\begin{array}{l}-0.002 \\
(0.003) \\
{[0.003]}\end{array}$ \\
\hline Education: Medium & $\begin{array}{c}-0.154^{* *} \\
(0.064) \\
{[0.059]}\end{array}$ & $\begin{array}{l}-0.004 \\
(0.052) \\
{[0.051]}\end{array}$ & $\begin{array}{c}0.003 \\
(0.058) \\
{[0.054]}\end{array}$ \\
\hline Education: High & $\begin{array}{l}-0.090 \\
(0.073) \\
{[0.076]}\end{array}$ & $\begin{array}{l}-0.007 \\
(0.056) \\
{[0.065]}\end{array}$ & $\begin{array}{c}0.026 \\
(0.069) \\
{[0.084]}\end{array}$ \\
\hline Cognitive Ability & & & $\begin{array}{l}{[0.001]} \\
-0.049 \\
(0.048) \\
{[0.057]}\end{array}$ \\
\hline Individuals & 926 & 926 & 746 \\
\hline $\mathrm{R}^{2}$ & 0.040 & 0.779 & 0.783 \\
\hline All Coefficients $=0$ (p-value $)$ & 0.000 & 0.993 & 0.820 \\
\hline District of arrival & no & yes & yes \\
\hline Year of arrival & no & yes & yes \\
\hline Country of origin & no & yes & yes \\
\hline
\end{tabular}

Note: The dependent variable is the network at migration. Standard errors in parenthesis: ${ }^{*} \mathrm{p}<0.10,{ }^{* *} \mathrm{p}<0.05,{ }^{* * *} \mathrm{p}<0.01$. We report standard errors clustered at district level in square brackets. 
Figure 1: Searching for a Job and Human Capital Investment

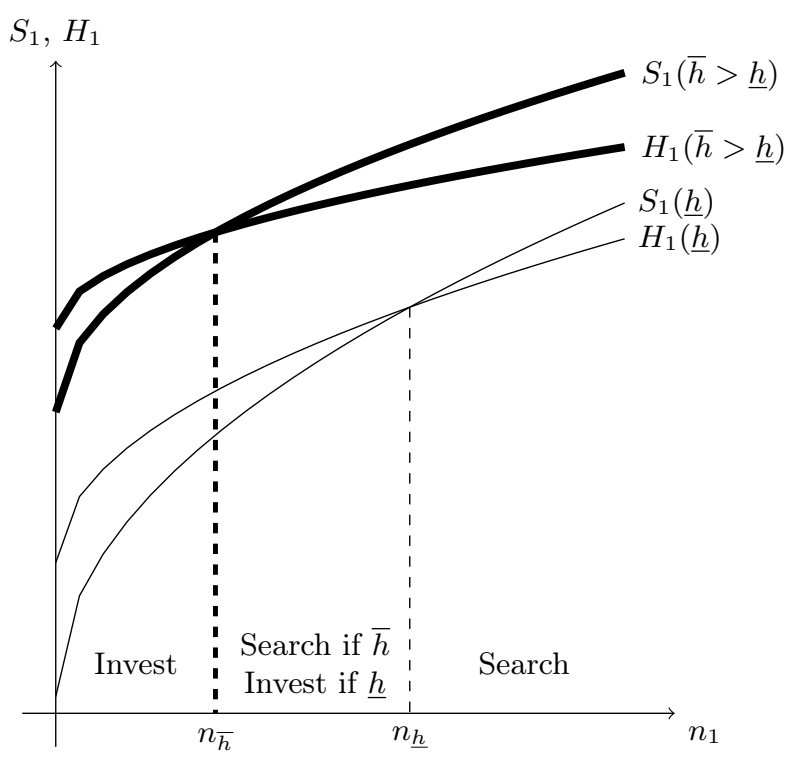

Figure 2: Wages at $t=2$ for a Given Level of Initial Human Capital $E\left(w_{2}\right) \mid \underline{h}$ conditional on working

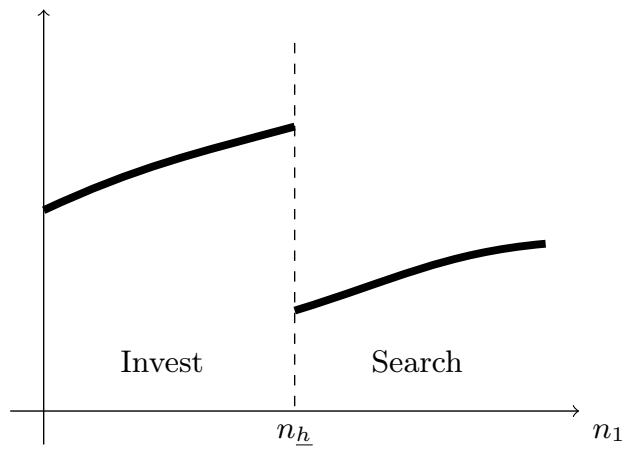


Figure 3: Effect of Network at Migration on Employment

Full Sample

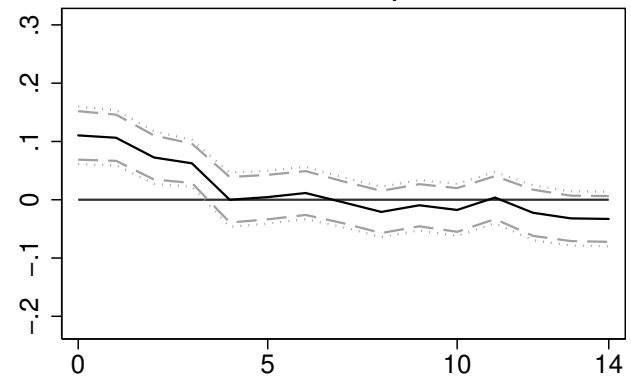

Medium Education

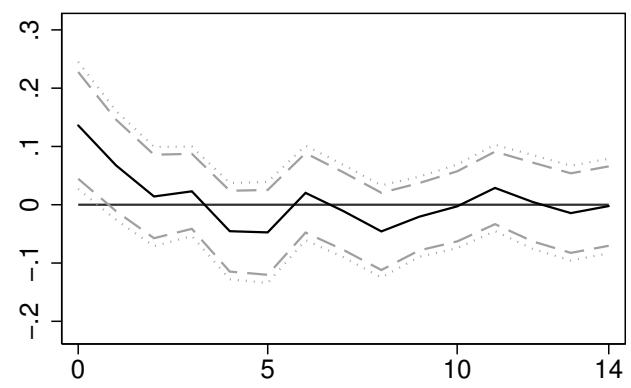

Low Education

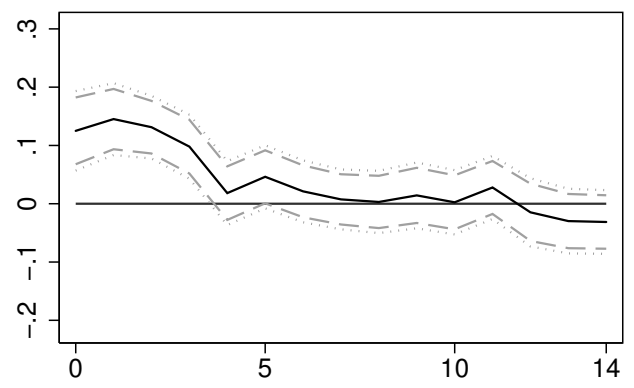

High Education

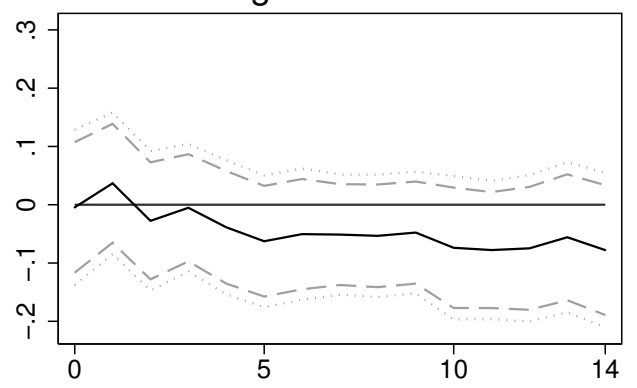

Note: the graphs report the coefficients of the network effect interacted with years since migration as well as confidence intervals ( 5 percent and 10 percent). The dependent variable is an indicator for employment defined as in Table 2. The estimated regression corresponds to specification (5), where we use yearly dummies for years since migration. Standard errors are clustered at individual level. Controls: gender, age and age at migration (and their squared), pre-migration controls, country, district and year at arrival fixed effects. 
Figure 4: Effect of Network at Migration on Human Capital Investment
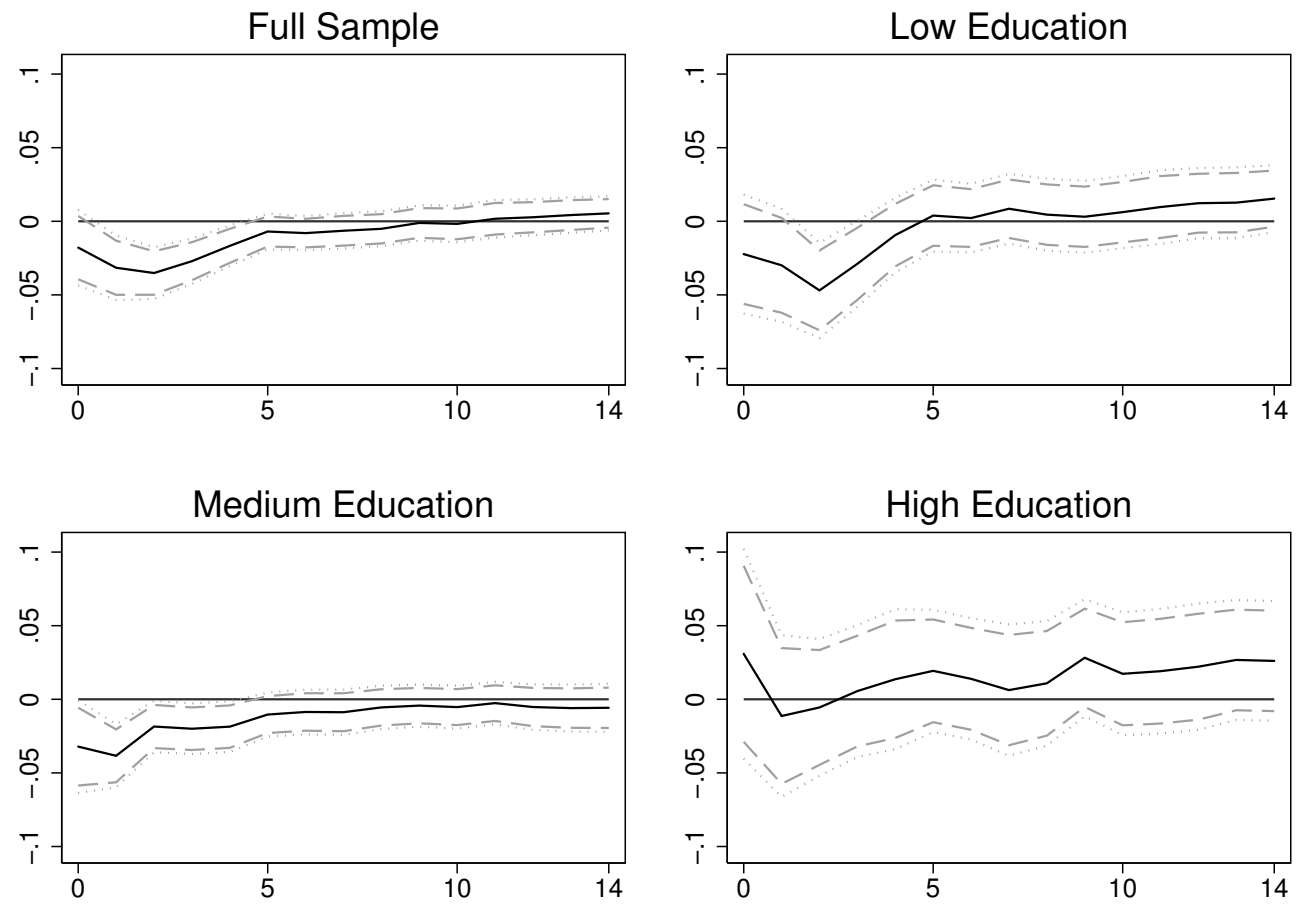

Note: the graphs report the coefficients of the network effect interacted with years since migration as well as confidence intervals ( 5 percent and 10 percent). The dependent variable is a indicator for being in education defined as in Table 3. The estimated regression corresponds to specification (5), where we use yearly dummies for years since migration. Standard errors are clustered at individual level. Controls: gender, age and age at migration (and their squared), pre-migration controls, country, district and year at arrival fixed effects. 
Figure 5: Effect of Network at Migration on (log) hourly Wage
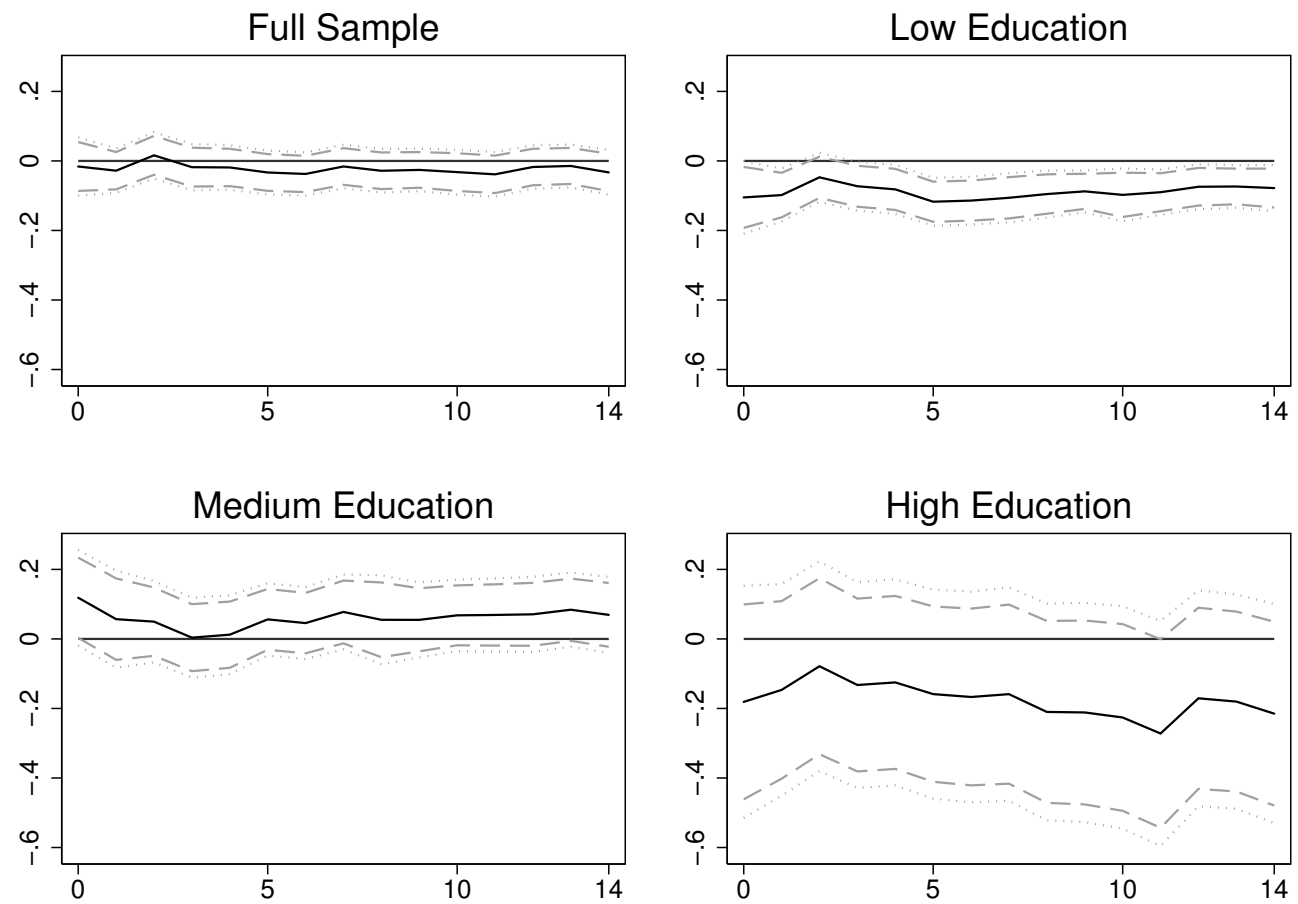

Note: the graphs report the coefficients of the network effect interacted with year since migration as well as confidence intervals ( 5 percent and 10 percent). The dependent variable is the (log) of real average hourly wages defined as in Table 5 . The estimated regression corresponds to specification (5), where we use yearly dummies for each year since migration. Standard errors are clustered at individual level. Controls: gender, age and age at migration (and their squared), pre-migration controls, country, district and year at arrival fixed effects. 


\section{References}

Åslund, Olof, Per-Anders Edin, Peter Fredriksson, and Hans Grönqvist (2011). Peers, Neighborhoods, and Immigrant Student Achievement: Evidence from a Placement Policy. American Economic Journal: Applied Economics 3(2), 67-95.

Bayer, Patrick, Stephen L. Ross, and Giorgio Topa (2008). Place of Work and Place of Residence: Informal Hiring Networks and Labor Market Outcomes. Journal of Political Economy 116(6), 1150-1196.

Beaman, Lori A. (2012). Social Networks and the Dynamics of Labour Market Outcomes: Evidence from Refugees Resettled in the U.S. The Review of Economic Studies 79(1), $128-161$.

Bennett, Patrick, Lisbeth La Cour, Birthe Larsen, and Gisela Waisman (2015). Negative Attitudes, Network and Education. Copenhagen Business School Working Paper 01-2015.

Bentolila, Samuel, Claudio Michelacci, and Javier Suarez (2010). Social Contacts and Occupational Choice. Economica 77(305), 20-45.

Bertrand, Marianne, Erzo F P Luttmer, and Sendhil Mullainathan (2000). Network effects and welfare cultures. The Quarterly Journal of Economics 115(3), 1019-1055.

Borjas, George J. (1998). To Ghetto or Not to Ghetto: Ethnicity and Residential Segregation. Journal of Urban Economics 44 (2), 228-253.

Calvó-Armengol, Antoni and Matthew O. Jackson (2004). The Effects of Social Networks on Employment and Inequality. American Economic Review 94 (3), 426-454.

Cutler, David M. and Edward L. Glaeser (1997). Are Ghettos Good or Bad? The Quarterly Journal of Economics 112(3), 827-872.

Damm, Anna Piil (2009). Ethnic Enclaves and Immigrant Labor Market Outcomes: QuasiExperimental Evidence. Journal of Labor Economics 27(2), 281-314.

Dustmann, Christian, Albrecht Glitz, Uta Schönberg, and Herbert Brücker (2016). Referralbased Job Search Networks. Review of Economic Studies 83(2), 514-546.

Dustmann, Christian and Ian Preston (2001). Attitudes to Ethic Minorities, Ethnic Context and Location Decisions. Economic Journal 111(470), 353-73.

Edin, Per-Anders, Peter Fredriksson, and Olof Åslund (2003). Ethnic Enclaves And The Economic Success Of Immigrants - Evidence From A Natural Experiment. The Quarterly Journal of Economics 118(1), 329-357.

Fitzenberger, Bernd, Aderonke Osikominu, and Robert Völter (2005). Imputation rules to improve the education variable in the IAB employment subsample. FDZ Methodenreport No. 3/2005.

Galenianos, Manolis (2013). Learning About Match Quality and the Use of Referrals. Review of Economic Dynamics 16(4), 668-690.

Galenianos, Manolis (2014). Hiring through referrals. Journal of Economic Theory 152(C), 304-323.

Glitz, Albrecht (2012). The Labor Market Impact of Immigration: A Quasi-Experiment Exploiting Immigrant Location Rules in Germany. Journal of Labor Economics 30(1), $175-213$.

Glitz, Albrecht (2014). Ethnic segregation in Germany. Labour Economics 29, 28-40.

Goel, Deepti and Kevin Lang (2009). Social Ties and the Job Search of Recent Immigrants. NBER Working Paper 15186.

Gould, Eric D., Victor Lavy, and M. Daniele Paserman (2009). Does Immigration Affect the Long-Term Educational Outcomes of Natives? Quasi-Experimental Evidence. Economic Journal 119(540), 1243-1269. 
Granovetter, Mark (1973). The Strength of Weak Ties. American Journal of Sociology 78(6), $1360-1380$.

Guryan, Jonathan, Kory Kroft, and Matthew J. Notowidigdo (2009). Peer Effects in the Workplace: Evidence from Random Groupings in Professional Golf Tournaments. American Economic Journal: Applied Economics 1(4), 34-68.

Hellerstein, Judith K., Melissa McInerney, and David Neumark (2011). Neighbors and Coworkers: The Importance of Residential Labor Market Networks. Journal of Labor Economics 29(4), 659-695.

Loury, Linda Datcher (2006). Some Contacts Are More Equal than Others: Informal Networks, Job Tenure, and Wages. Journal of Labor Economics 24(2), 299-318.

Montgomery, James D (1991). Social Networks and Labor-Market Outcomes: Toward an Economic Analysis. The American Economic Review 81(5), 1408-1418.

Patacchini, Eleonora and Yves Zenou (2012). Ethnic networks and employment outcomes. Regional Science and Urban Economics 42(6), 938-949.

Pellizzari, Michele (2010). Do Friends and Relatives Really Help in Getting a Good Job? Industrial and Labor Relations Review 63(3), 494-510.

Regets, Mark C. and Harriet Orcutt Duleep (1999). Immigrants and Human-Capital Investment. American Economic Review 89(2), 186-191.

Schmutte, Ian M (2015). Job referral networks and the determination of earnings in local labor markets. Journal of Labor Economics 33(1), 1-32.

van den Berg, Gerard J and Bas van der Klaauw (2006). Counseling And Monitoring Of Unemployed Workers: Theory And Evidence From A Controlled Social Experiment. International Economic Review 47(3), 895-936.

Xie, Yu and Margaret Gough (2011). Ethnic Enclaves and the Earnings of Immigrants. Demography 48(4), 1293-1315. 


\section{Appendices for Online Publication}

\section{A Analytical Discussion of Propositions}

In Section 3, we discuss three Prepositions with the three main implications of our simple model, which we compare to what we find in our empirical exercise. In this short Appendix we present a slightly more structured discussion as a way to motivate those prepositions. We first focus on Proposition 2, which concerns the effects of a changing level of $n_{1}$ on the choice of our agent between searching and human capital investment, taking $h_{1}$ as given. Here we will therefore look at the derivatives with respect to $n$, and then look at the effects of changing $h$ on outcomes as a comparative statics exercise. In order to evaluate the effects of initial network size on the $t=1$ choice between human capital investment $\left(H_{1}\right)$ and searching for a job $\left(S_{1}\right)$, lets us look at the first derivative of these functions with respect to $n$.

$$
\frac{\partial H_{1}}{\partial n_{1}}=\beta\left(\frac{\partial \mathcal{S}\left(h^{\prime}\right)}{\partial n_{1}}\right)
$$

Let us now look at the first derivative of the $S_{1}$ function with respect to $n_{1}$.

$$
\frac{\partial S_{1}}{\partial n_{1}}=(1+\beta)\left(\frac{\partial \mathcal{S}(\bar{h})}{\partial n_{1}}\right)
$$

In order to sign the above two derivatives we need to sign $\frac{\partial \mathcal{S}(h)}{\partial n_{1}}$, the next step. In order to mantain relatively compact notation, let us define:

$$
\begin{aligned}
\int \max \left\{x_{i}-b_{u}, 0\right\} d F_{i}\left(x_{i}\right) & \equiv \mathcal{A} \\
\int \max \left\{x_{f}-b_{u}, 0\right\} d F_{f}\left(x_{f}\right) & \equiv \mathcal{B} \\
\int \max \left\{x_{i}-b_{u}, x_{f}-b_{u}, 0\right\} d F_{i}\left(x_{i}\right) d F_{f}\left(x_{f}\right) & \equiv \mathcal{C}
\end{aligned}
$$

We can now write

$$
\begin{aligned}
\frac{\partial \mathcal{S}(h)}{\partial n} & =\frac{\partial p_{i}}{\partial n}\left(1-p_{f}(h)\right) \mathcal{A}-p_{f}(h) \frac{\partial p_{i}}{\partial n} \mathcal{B}+\frac{\partial p_{i}}{\partial n} p_{f}(h) \mathcal{C} \\
& =\frac{\partial p_{i}}{\partial n}\left[\left(1-p_{f}(h)\right) \mathcal{A}+p_{f}(h)(\mathcal{C}-\mathcal{B})\right]
\end{aligned}
$$


Assuming that these two distributions have common support, $\mathcal{C}>\mathcal{B}$ (if the common support assumption does not hold, this equation would hold as weak inequality). In other words, the value of drawing twice from two distributions that partially overlap and picking the better outcome is strictly better (in expectations) than drawing from one of those distributions only. Therefore, $\frac{\partial \mathcal{S}(h)}{\partial n}>0$. This implies that both the value of searching and the value of human capital investment increase in $n_{1}$. The outcome from searching is positively affected by networks, which makes it more likely to find a job and increase the expected wage (because of the increased probability of drawing two offers and picking the higher one in that case).

What can help us understand how $n_{1}$ affects our individual's decision is to compare these two derivatives. We therefore compare $\frac{\partial \mathcal{S}\left(h^{\prime}\right)}{\partial n_{1}}$ with $\frac{\partial \mathcal{S}(\bar{h})}{\partial n_{1}}$ where $h^{\prime}>\bar{h}$. Let us write the cross derivative of $\mathcal{S}$ with respect to $n$ and $h$ as

$$
\begin{aligned}
\frac{\partial^{2} \mathcal{S}(h)}{\partial n \partial h} & =-\frac{\partial p_{i}}{\partial n} \frac{\partial p_{f}}{\partial h} \mathcal{A}-\frac{\partial p_{i}}{\partial n} \frac{\partial p_{f}}{\partial h} \mathcal{B}+\frac{\partial p_{i}}{\partial n} \frac{\partial p_{f}}{\partial h} \mathcal{C} \\
& =\frac{\partial p_{i}}{\partial n} \frac{\partial p_{f}}{\partial h}(\mathcal{C}-\mathcal{A}-\mathcal{B})
\end{aligned}
$$

Under our assumption that both wage distributions have only non negative outcomes $\mathcal{C}<\mathcal{A}+\mathcal{B}$. This directly implies that $\frac{\partial \mathcal{S}^{2}(h)}{\partial n \partial h}<0$, which in turn means that $\frac{\partial \mathcal{S}\left(h^{\prime}\right)}{\partial n}<$ $\frac{\partial \mathcal{S}(\bar{h})}{\partial n}$. In words, when individuals have high levels of human capital in our setup, the marginal effect of network size on search outcomes is (positive but) smaller. Since $\beta>0$, this means that $\frac{\partial \mathcal{S}}{\partial n}>\frac{\partial H_{1}}{\partial n}$. This result, together with the results on second derivative we move to next, helps us analyze the comparative statics of the choice outlined by our model intuitively and graphically.

Having established that both the value of human capital investment and the value of searching for a job are monotonically increasing in $n_{1}$ for a given level of $h_{1}$, we next look at the second derivative of the same two functions.

$$
\begin{gathered}
\frac{\partial^{2} H_{1}}{\partial n^{2}}=\beta\left(\frac{\partial^{2} \mathcal{S}\left(h^{\prime}\right)}{\partial n^{2}}\right) \\
\frac{\partial^{2} S_{1}}{\partial n^{2}}=(1+\beta)\left(\frac{\partial^{2} \mathcal{S}(\bar{h})}{\partial n^{2}}\right)
\end{gathered}
$$

In order to sign these derivatives and compare them, we need to sign the terms in brackets. We evaluate that term for a generic $h$ so that we can investigate how it is 
affected by the level of $h$.

$$
\begin{aligned}
\frac{\partial^{2} \mathcal{S}(h)}{\partial n^{2}} & =\frac{\partial^{2} p_{i}}{\partial n^{2}}\left(1-p_{f}(h)\right) \mathcal{A}-p_{f}(h) \frac{\partial^{2} p_{i}}{\partial n^{2}} \mathcal{B}+\frac{\partial^{2} p_{i}}{\partial n^{2}} p_{f}(h) \mathcal{C} \\
& =\frac{\partial^{2} p_{i}}{\partial n^{2}}\left(1-p_{f}(h)\right) \mathcal{A}+\frac{\partial^{2} p_{i}}{\partial n^{2}} p_{f}(h)(\mathcal{C}-\mathcal{B})<0
\end{aligned}
$$

since $\mathcal{C}>\mathcal{B}$. So both our value functions (human capital investment and search, given initial human capital level) have negative second derivatives.

In order to accurately draw those functions and in particular to evaluate whether the single-crossing result holds, it is useful to look at whether and how the magnitude of this second derivative depends on $h$. It is useful to rewrite the equation above as

$$
\frac{\partial^{2} \mathcal{S}(h)}{\partial n^{2}}=\frac{\partial^{2} p_{i}}{\partial n^{2}} \mathcal{A}+\frac{\partial^{2} p_{i}}{\partial n^{2}}(\mathcal{C}-\mathcal{A}-\mathcal{B}) p_{f}(h)
$$

The first term of equation (16) above is unaffected by $h$. The second term is positive because $\frac{\partial^{2} p_{i}}{\partial n^{2}}<0$ and $\mathcal{C}-\mathcal{A}-\mathcal{B}<0$. Since $\frac{\partial p_{f}(h)}{\partial h}>0$, the overall derivative is increasing in $h$ (it is less negative for larger values of $h$ ). We can now compare the second derivatives of the human capital investment and job searching functions:

$$
\begin{aligned}
\frac{\partial^{2} S\left(h^{\prime}\right)}{\partial n^{2}}>\frac{\partial^{2} S(\bar{h})}{\partial n^{2}} \\
\beta \frac{\partial^{2} S\left(h^{\prime}\right)}{\partial n^{2}}>(1+\beta) \frac{\partial^{2} S(\bar{h})}{\partial n^{2}}
\end{aligned}
$$

where the second line follows from the fact that both second derivatives are negatives and $\beta$ is positive. Therefore,

$$
\frac{\partial^{2} H_{1}}{\partial n^{2}}>\frac{\partial^{2} S_{1}}{\partial n^{2}}
$$

In words, we found that both the value function for human capital investment and for job search are monotonically increasing concave functions of network size (for a given level of human capital initial endowment), and that both first and second derivatives are smaller (in absolute value) for the human capital investment function. With the information above, we can draw the $S_{1}$ and the $H_{1}$ curves on a chart, which lets us evaluate the content of Propositions 1 and 2. To the left of the intersection point $n_{\underline{h}}$ in Figure A.1 the individual will decide to invest in human capital, because it give higher utility in expectations. To the right of $n_{\underline{h}}$ (i.e. for larger size of initial social network) she will decide to search for a job. Figure A.1 is drawn for a certain 
Figure A.1: Value of search and of human capital investment

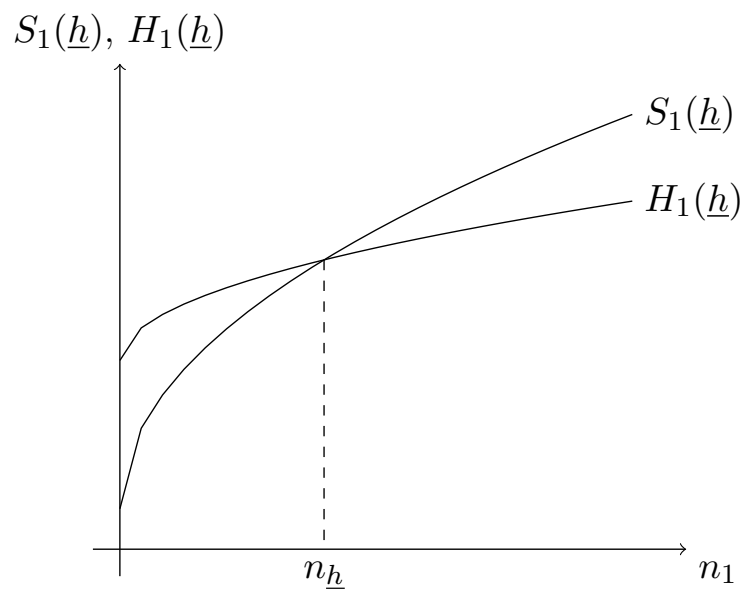

human capital $\underline{h}$. However, the relative position of the two curves depend on the level of initial human capital and on other parameters. For example, under certain parameter configurations there may be a corner solution where $h$ is sufficiently high that the the value of search is higher than the value of human capital investment even on the vertical axis. In this case, the agent will decide to look for a job at $t=1$ for all levels of $n_{1}$. From Figure A.1 above we can also investigate the effects of changing $h_{1}$ on the equilibrium values. Since each curve on the chart above is drawn for a certain level of $h$, curves will shift as we let $h$ vary. Symmetrically to our analysis for $n$ above, it is very easy to show that

$$
\frac{\partial S_{1}}{\partial h}>\frac{\partial H_{1}}{\partial h}
$$

while both derivatives are positive, the $S_{1}$ curve reacts more strongly than the $H_{1}$ curve to changes in $h$. Looking back at Figure A.1, as we increase $h$ the equilibrium value of $n$ that will make our individual indifferent between searching for a job and acquiring human capital at $t=1$ will be lower. If we compare two individuals with the same social network but with different levels of initial human capital, the individual with higher initial human capital is more likely to start looking for a job earlier. Based on equation (12) above, at higher levels of $h$ the effect of network on both curves is smaller is magnitude, and therefore equilibrium values respond less to changes in $n$ at higher levels of $h$, which is what Proposition 3 states. 


\section{B Institutional Background: Random Allocation of Asy- lum Seekers and Ethnic Germans}

The allocation of asylum seekers is regulated at Federal level by the Asylum Procedure Act (Asylverfahrensgesetz). According to the law asylum seekers are allocated first to initial reception facilities and have no freedom to move. Each Federal State is assigned a defined quota (Königsteiner Schlüssel), which is calculated every year according to the tax receipts and population of the State. Consideration is also given to whether the branch office of the Federal Office responsible for the reception center deals with the asylum seeker's home country. After the first period in reception facilities, which can last up to a maximum of six months, the asylum seekers are placed in a district within the state of the first allocation. The state authorities decide whether to place them in collective accommodations, or whether to grant the applicant a permit to take an apartment. This discretionary decision must take account of both the public interest and the asylum seeker's personal concerns. The residence obligation ends as soon as the Federal Office grants asylum or refugee status. In special cases, for example in cases of family reunification, asylum seekers may also be assigned to a different reception center at their own request. The average duration of the application procedure was around two years as of 2010.

In case of ethnic Germans the allocation was regulated by the Residence Allocation Act (Wohnortzuweisungsgesetz). Starting from July 1989, ethnic Germans were granted a German visa upon application, and registered with a central authority. Those without a job (which comprised the vast majority of them) were distributed to one of the 16 federal states according to pre-specified state quotas. The further allocation to districts was regulated within each federal state, according to state-specific allocation. In some cases, the applicant could ask to join the relatives already living in the country. However, since the constraint of residence restriction turned out not to be enforced, a new law was passed in March 1996, which made the restriction stricter. Those who moved from the assigned district lost access to welfare benefits. The law was then abolished in December 2009. In our analysis we select only ethnic Germans entering Germany between 1996 and 2009, when the restriction on residence was stricter (see Glitz, 2012). 


\section{Survey Data linked to Administrative records}

The IAB-SOEP Migration Sample is a new longitudinal survey of individuals with migration background in Germany. The survey is carried out jointly by the Institute for Employment Research (IAB) and the German Socio Economic Panel (GSOEP). The survey has a panel structure, the first wave was carried out in 2013 and the second wave in 2014. The starting sample consisted of around 5,000 individuals. Part of the original sample (the head of household) was drawn from the German Social Security Archive (IEB), therefore the head of households are individuals who have been at least once part of the labor force, registered as unemployed, or benefits recipient. All family members were also interviewed. A subsample of the original survey sample has been linked to the social security data (IEB), using a personal identifier. At the end of the questionnaire, due to data protection, respondents are asked to give their consent for the record linkage. The overall approval rate amounts to around 50 percent. The final linked sample consists of 2,089 individuals: 2,028 from the first wave and 61 from the second wave. Our sample consists of 933 individuals: 922 from the first wave and 17 from the second wave. This final sample is obtained excluding second generation migrants, those with missing information in the variables of interest, and those entering as student or still in education at the time of the survey.

\section{Additional Tables}

Table D.1: Comparing Refugees and non Refugees in Survey Data (share)

\begin{tabular}{lccc}
\hline \hline & Refugees & Not Refugees & P-value \\
\hline Pre Migration Employment & 0.519 & 0.442 & 0.001 \\
Pre Migration Edu: Low & 0.586 & 0.700 & 0.000 \\
Pre Migration Edu: Medium & 0.241 & 0.154 & 0.000 \\
Pre Migration Edu: High & 0.174 & 0.146 & 0.119 \\
Pre Migration Language: Good & 0.130 & 0.060 & 0.000 \\
Current Language: Good & 0.581 & 0.473 & 0.000 \\
\hline Source: IAB/SOEP Migration Sample (first and second wave). P-value: & significant \\
difference between two samples. & &
\end{tabular}

difference between two samples. 
Table D.2: Employment and Human Capital Investment Over Time (share)

\begin{tabular}{lcc}
\hline \hline Years since Migration & Work & In Education \\
\cline { 2 - 3 } $0-2$ & 0.480 & 0.119 \\
$3-5$ & 0.717 & 0.040 \\
$6-9$ & 0.757 & 0.020 \\
$10+$ & 0.763 & 0.011 \\
\hline Total & 0.688 & 0.044 \\
\hline
\end{tabular}

Note: Data sources: IAB-SOEP Migration Sample linked to IEB data.

Table D.3: Network at Migration and Methods of Finding the First Job in Germany

\begin{tabular}{lccc}
\hline \hline \multirow{2}{*}{ Method } & Contacts & Internet & Empl. Agency \\
\cline { 2 - 4 } & $(1)$ & $(2)$ & $(3)$ \\
\hline Low Edu & 0.090 & -0.039 & -0.004 \\
& $(0.062)$ & $(0.043)$ & $(0.041)$ \\
Netw $_{t 0}$ (a) & -0.008 & -0.034 & 0.033 \\
Netw $_{t 0}$ xLow Edu & $(0.054)$ & $(0.043)$ & $(0.034)$ \\
& $0.098^{* *}$ & -0.043 & -0.041 \\
Individuals $_{\text {R }}^{2}$ & $(0.045)$ & $(0.037)$ & $(0.030)$ \\
(a)+(b)==0 (p-value) & 799 & 799 & 799 \\
\hline Mean Dep. Var & 0.428 & 0.362 & 0.404 \\
\hline
\end{tabular}

Note: The dependent variable is an indicator for different methods of finding the first job in Germany, the method varying according to the heading. All network variables are standardised: the relevant coefficient corresponds to the effect of an increase by one standard deviation. Controls include base controls and pre-migration controls. Base controls: gender, age at migration (and its sq.), country of origin fixed effects, year at migration fixed effects. Pre-migration controls: employment, language proficiency, education, working experience. Standard errors in parenthesis are clustered at district level: ${ }^{*} \mathrm{p}<0.10$, language proficiency, ed
$* * \mathrm{p}<0.05, * * * \mathrm{p}<0.01$

Table D.4: Robustness. Control for Cognitive Skills

\begin{tabular}{|c|c|c|c|c|}
\hline \multirow[t]{2}{*}{ Dependent variable: } & \multicolumn{2}{|c|}{ Employment } & \multicolumn{2}{|c|}{ Human Capital } \\
\hline & (1) & $(2)$ & (3) & (4) \\
\hline Netw $_{t 0}$ & $\begin{array}{c}0.105^{* * *} \\
(0.021)\end{array}$ & $\begin{array}{c}0.094^{* * *} \\
(0.023)\end{array}$ & $\begin{array}{c}-0.028^{* * *} \\
(0.009)\end{array}$ & $\begin{array}{c}-0.029 * * * \\
(0.010)\end{array}$ \\
\hline Netw $_{t 0} \times Y_{s m} 3-5$ & $\begin{array}{c}-0.074^{* * *} \\
(0.016)\end{array}$ & $\begin{array}{c}-0.081 * * * \\
(0.017)\end{array}$ & $\begin{array}{c}0.011 \\
(0.009)\end{array}$ & $\begin{array}{c}0.009 \\
(0.009)\end{array}$ \\
\hline Netw $_{t 0} \times Y_{s m 6}+$ & $\begin{array}{c}-0.098^{* * *} \\
(0.017)\end{array}$ & $\begin{array}{c}-0.095^{* * *} \\
(0.017)\end{array}$ & $\begin{array}{c}0.031^{* * *} \\
(0.009)\end{array}$ & $\begin{array}{c}0.031^{* * * *} \\
(0.010)\end{array}$ \\
\hline Cognitive Skills & & $\begin{array}{c}0.019 \\
(0.021) \\
\end{array}$ & & $\begin{array}{c}0.004 \\
(0.009) \\
\end{array}$ \\
\hline Individuals & 926 & 752 & 926 & 746 \\
\hline Obs & 12132 & 9782 & 12094 & 9660 \\
\hline $\mathrm{R}^{2}$ & 0.236 & 0.246 & 0.209 & 0.215 \\
\hline
\end{tabular}




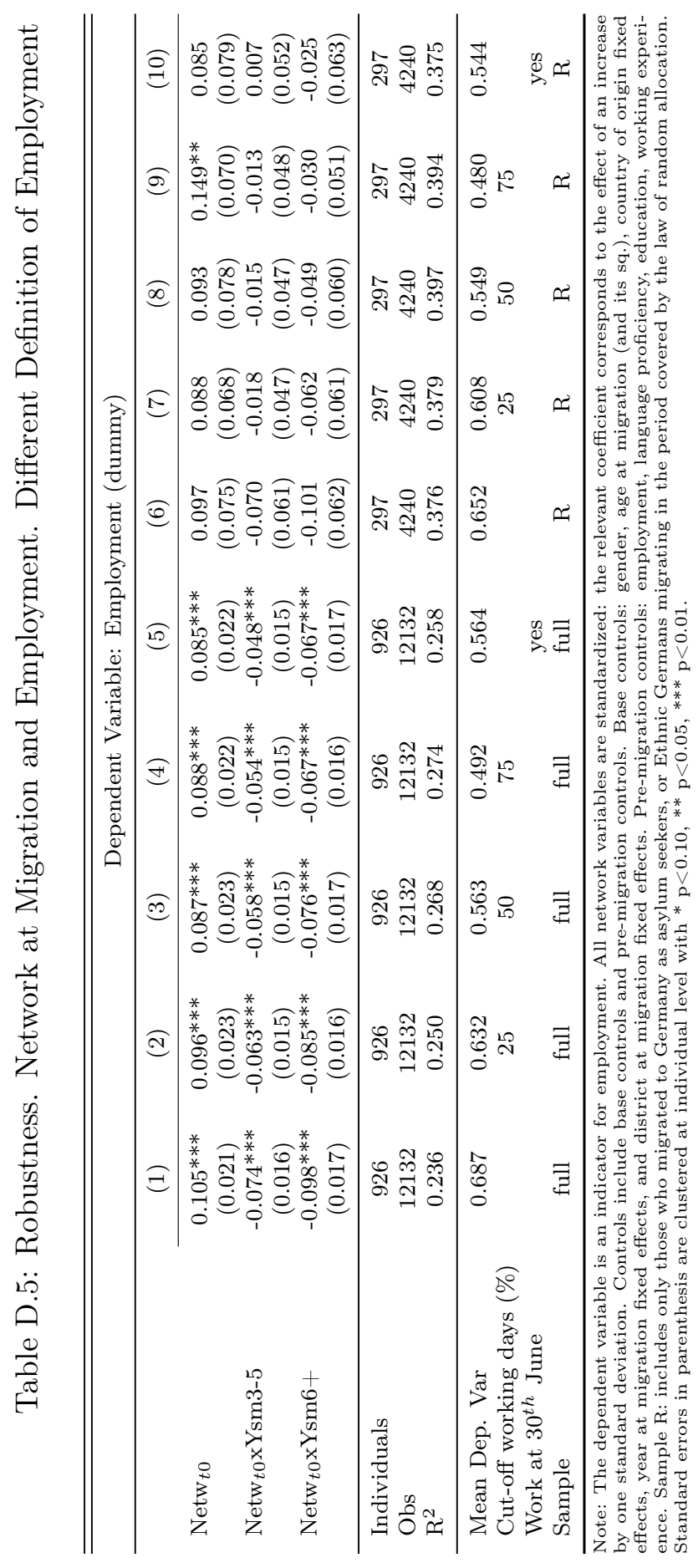




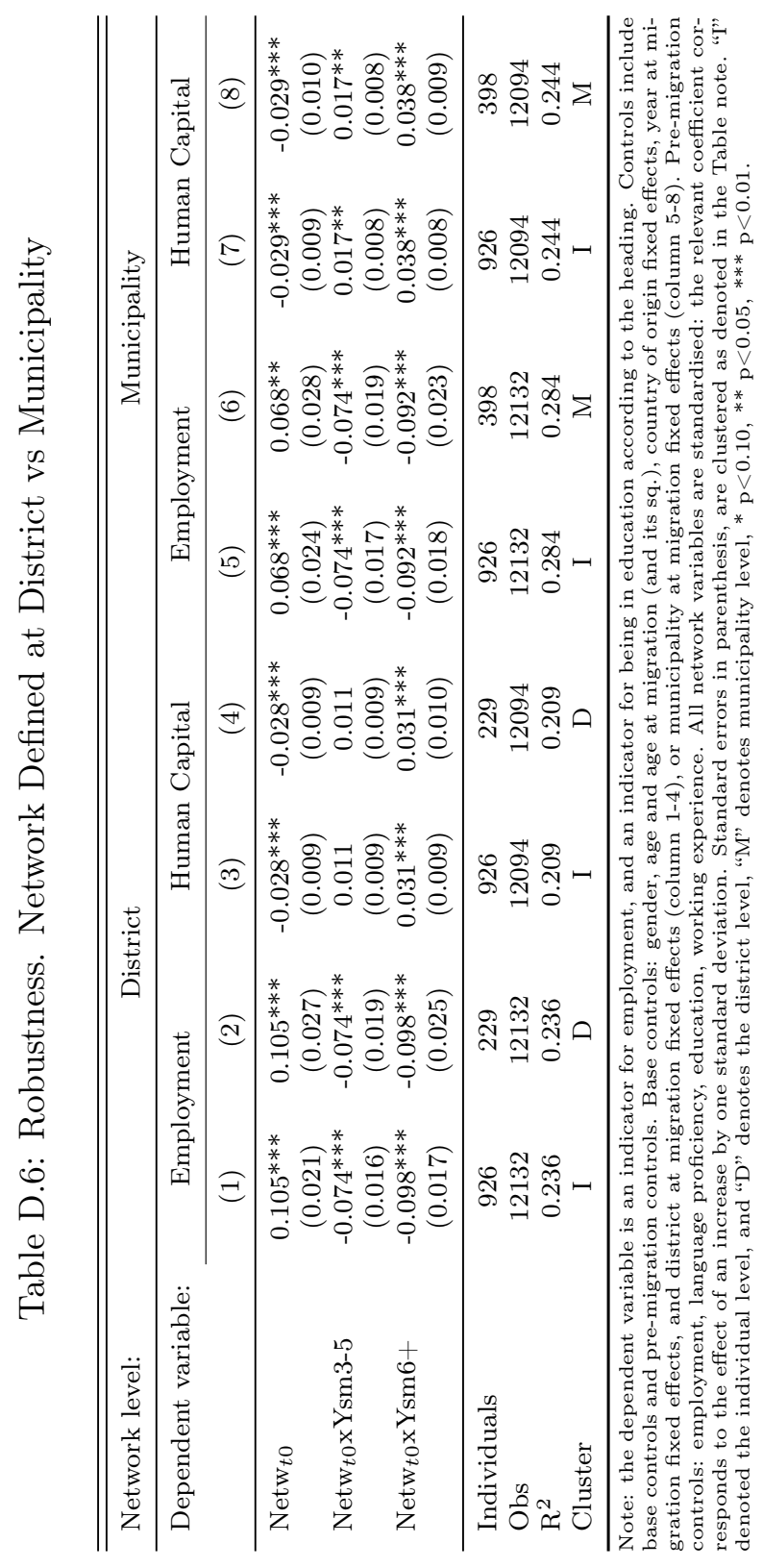

